\title{
Fermion mass mixing and vacuum triviality in the renormalization group procedure for effective particles
}

\author{
Stanisław D. Głazek \\ Institute of Theoretical Physics, Faculty of Physics, University of Warsaw
}

(Dated: 15 May, 2013)

\begin{abstract}
Renormalization group procedure for effective particles is applied to the model quantum theory of free fermions to which one adds an interaction in the form of a mass mixing term. If one used a standard approach based on the instant form of dynamics, the theory would suffer from a generic vacuum problem caused by a divergent production of virtual quanta out of a bare vacuum and it would require an adjustment of its degrees of freedom to the added interaction term before quantization, considered a means of avoiding the quantum vacuum problem. In the effective particle approach, the quantum vacuum problem is dealt with instead by using the front form of dynamics, where the pair production is excluded by momentum conservation. The corresponding Hamiltonian includes mass parameters through constraint equations while the required quantum field operators are constructed independently of all mass parameters, including the parameters that appear in the added mass mixing interaction term. Then the masses and states of physical fermions emerge at an end of the non-perturbative calculation that is carried out entirely in one and the same interacting quantum theory with a trivial vacuum and no quantization adjustment. An a priori infinite set of renormalization group equations for all momentum modes of fermion fields is reduced to just one equation for a two-by-two mass squared matrix, thanks to 7 kinematical symmetries of the front form (the instant form has only 6). For strong mass mixing interactions, the fermion model solutions qualitatively differ from the analogous, earlier found boson model solutions by the absence of tachyons.
\end{abstract}

PACS numbers: 11.10.Gh, 11.10.Hi, 11.30.Cp

\section{INTRODUCTION}

It has been recently shown [1] that a theory of quantum scalar fields with mass mixing interactions can be solved non-perturbatively using the renormalization group procedure for effective particles (RGPEP). This article shows that the RGPEP can also solve a quantum theory of fermion fields with arbitrarily strong mass mixing interactions. Thus, RGPEP is found to pass the test of solving elementary theories beyond perturbative expansions. For example, the procedure demonstrates that the fermion theories do not have tachyon solutions no matter how strong the mass-mixing interactions are, in distinction from the boson theories that have tachyon solutions for sufficiently strong mass-mixing interactions.

The theories of fermions or bosons with mass mixing interactions can be constructed using different forms of dynamics [2]. The most commonly used form of dynamics is called the instant form (IF), where the evolution of a system is traced from one time to another. Both the boson and the fermion theories exhibit an ultraviolet divergent vacuum problem in their IF versions. The problem is caused by a copious creation of virtual particle pairs of unlimited virtuality [3]. The only IF method that the author knows for circumventing the vacuum problem caused by mass mixing is to return to a classical theory and to change the quantization procedure in a way that depends on the mass-mixing interaction. This method is called here re-quantization. Unfortunately, it is not clear how to apply the method of re-quantization to theories of great physical interest, such as QCD or the electro-weak theory with massive neutrinos. The reason is that the corresponding relativistic quantum interactions are not sufficiently understood to establish if there exist some classical degrees of freedom that are suitable for the purpose.

The RGPEP is systematically applied to a quantum theory with mass mixing interactions without any need for re-quantization. The vacuum problem is avoided in the RGPEP by using an alternative form of dynamics to the IF. The alternative form is called the front form (FF) [2]. In the FF, the evolution of a system is traced from one value of $t+z$ to another. The creation of pairs out of the bare vacuum by a translation invariant interaction, which by necessity conserves momentum, is not possible in a regulated FF theory since the pairs must carry a non-zero kinematical momentum while the vacuum carries zero. Moreover, the FF has 7 kinematical symmetries instead of only 6 in the IF and the RGPEP preserves these 7 symmetries. The symmetries result in a reduction of an infinite set of differential renormalization group equations for all Fourier components of quantum fields to just one $2 \times 2$ matrix equation for masses of effective particles. This is not a mere computational simplification because the reduction of solving a mass-mixing theory to solving just two coupled RGPEP equations for the masses of effective particles allows one to avoid the issue of regulating the theory in ultra-violet and subsequently removing the ultra-violet cutoff dependence from observables, in order to recover the Lorentz symmetry in the spectrum of solutions (see below).

Some details of construction of quantum fermion fields in the RGPEP are worth mentioning because they are 
helpful in handling the FF constraint equations and the Lorentz symmetry. In distinction from the IF quantum fermion fields, the unconstrained parts of the FF quantum fermion fields have only two components instead of four and these two are constructed purely kinematically in terms of their Fourier components. Hence, the unconstrained quantum fields do not depend on the fermion mass parameters. These parameters only enter in the Hamiltonian as coefficients of products of the quantum fields, as a consequence of the constraint equations. Thus, the FF construction of quantum fermion field operators avoids the IF difficulties due to assigning masses to fermions as if they were free while the Hamiltonian includes interactions. More generally, the little group [4] that preserves the front allows one to build states and operators for fermions with arbitrary kinematical momenta irrespective of the interaction. Such purely kinematical construction of quantum field operators is not possible in the IF theory. The reason is that the motion of fermions in the IF requires a spinor representation of the Lorentz boosts. A priori, the boost generators depend on interactions and the simplest form of such dependence occurs through the mass terms. This is also why the IF Fourier expansion of quantum fermion fields depends on the fermion masses.

Comprehensive description of the RGPEP for fermions requires several elements that are collected in several Appendices, in order to avoid crowding the main text with details. However, the main text does include the details that concern basic features of quantum field theory, a subject of a long history [5, [6] and unyielding relevance [7]. The only details that are not discussed comprehensively concern regularization of fermion fields. These details are not required for completeness of the article because the RGPEP equations turn out to reduce to two equations for mass parameters only and these equations are entirely independent of particle momenta in our mass mixing models (this is a consequence of a general design of the RGPEP). Thus all regularizations based on limiting a momentum range in the Fourier expansion of quantum fields are of no consequence for the obtained solutions and the solutions satisfy all requirements of special relativity and quantum mechanics within any finite range of momentum under consideration.

So, the article point is not just that the RGPEP can be used to solve a simple theory, but that it is defined in quite general terms and works well in the test case with fermions, in addition to the test it had passed earlier for bosons [1]. Of course, solutions of the RGPEP equations in complex theories cannot be found as easily as in the simple models with mass mixing. Nevertheless, one may hope that the RGPEP will help in searches for feasible ways of constructing numerical approximations to solutions of complex relativistic theories, such as the FF version of QCD [8].

The article is organized as follows. Section [I]describes the model theory of fermions with mass mixing interaction terms in the standard approach based on the IF of dynamics. One starts with constructing a quantum theory of free fermions, adds mass mixing interactions, discovers the divergent vacuum problem, and goes back to the classical theory in order to re-quantize it using new fields and thus get rid of the vacuum problem. The outcome is an expectation of how the solution to the quantum theory of fermions with mass mixing interactions could look like. Section III describes the FF approach. Once the FF quantum theory is defined, by constructing the unconstrained quantum field operators kinematically and taking into account the constraint equations in constructing the FF Hamiltonian, the vacuum problem is absent because the interaction terms do not create pairs from the bare vacuum state. The RGPEP procedure is then applied to solving the quantum theory without any need for re-quantization. The procedure leaves the trivial vacuum state unchanged. At the end of the RGPEP, one arrives at the same spectrum of solutions as the one expected on the basis of re-quantization in the IF. Sec. IV concludes the article by an explanation of a qualitative difference between the fermion and boson models concerning tachyons when the mass mixing interactions are strong. Appendix $\mathrm{A}$ describes a representation of $\gamma$ matrices that is useful in constructing FF theories. Kinematical construction of the FF quantum fermion fields is described in Appendix B. For completeness, Appendix C recapitulates elements of the RGPEP in general terms. Explicit solutions of the RGPEP equations in the fermion model are described in Appendix D.

\section{IF THEORY OF MASS MIXING}

We start our discussion with a brief recollection of the commonly known IF theory of free fermion fields. For simplicity, we explicitly consider just two fields. The quantum theory is obtained by imposing anticommutation relations on the fields. Then we add the mass mixing interaction term to the free Hamiltonian and thus obtain an elementary example of the Dirac vacuum problem [3]. The problem is then dealt with by going back to a classical theory and introducing two new fermion fields for which the classical Lagrangian density does not contain mass mixing terms. The FF approach based on the RGPEP will be shown in Sec. III to be different.

\section{A. IF free fermions}

Consider the Lagrangian density,

$$
\mathcal{L}=\bar{\psi}(i \not \partial-\mu) \psi+\bar{\phi}(i \not \partial-\nu) \phi,
$$

for two types of fermion fields $\psi$ and $\phi$ with masses $\mu$ and $\nu$. Variation of the action $A=\int d^{4} x \mathcal{L}$ with respect to $\bar{\psi}$ and $\bar{\phi}$ yields the Dirac equations of motion

$$
(i \not \partial-\mu) \psi=0
$$




$$
(i \not \partial-\nu) \phi=0 .
$$

The corresponding IF Hamiltonian has the form

$$
H=\int d^{3} x \mathcal{T}^{00},
$$

where $\mathcal{T}^{00}=\mathcal{H}$ denotes the energy density, i.e., the $\rho=\sigma=0$ component of the energy-momentum density tensor

$$
\mathcal{T}^{\rho \sigma}=\frac{\partial \mathcal{L}}{\partial \partial_{\rho} \psi_{\alpha}} \partial^{\sigma} \psi_{\alpha}+\frac{\partial \mathcal{L}}{\partial \partial_{\rho} \phi_{\alpha}} \partial^{\sigma} \phi_{\alpha}-g^{\rho \sigma} \mathcal{L} .
$$

The resulting Hamiltonian reads

$$
H=\int d^{3} x\left[\psi^{\dagger}(i \vec{\alpha} \vec{\partial}+\beta \mu) \psi+\phi^{\dagger}(i \vec{\alpha} \vec{\partial}+\beta \nu) \phi\right] \text {. }
$$

For the purpose of constructing a quantum theory, the Fourier decomposition of the fields at $x^{0}=0$ is arranged in the forms

$$
\begin{aligned}
\psi(\vec{x}) & =\sum_{\mu p s}\left[u_{\mu p s} b_{\mu p s} e^{i \vec{p} \vec{x}}+v_{\mu p s} d_{\mu p s}^{\dagger} e^{-i \vec{p} \vec{x}}\right], \\
\phi(\vec{x}) & =\sum_{\nu p s}\left[u_{\nu p s} b_{\nu p s} e^{i \vec{p} \vec{x}}+v_{\nu p s} d_{\nu p s}^{\dagger} e^{-i \vec{p} \vec{x}}\right] .
\end{aligned}
$$

We explicitly explain the notation for the field $\psi$. Notation for $\phi$ is obtained by replacing the mass $\mu$ with $\nu$.

The meaning of summing over spins and integrating over momentum is defined by

$$
\sum_{\mu p s}=\sum_{s= \pm 1} \int \frac{d^{3} p}{(2 \pi)^{3} 2 E_{\mu p}}
$$

$E_{\mu p}=\sqrt{\mu^{2}+\vec{p}^{2}}$, etc. The subscript $\mu$ refers to the dependence on the mass parameter. The spinors are obtained by boosting spinors at rest (cf. Ref. [9], Chap. $3)$,

$$
\begin{aligned}
& u_{\mu p s}=B(\mu, \vec{p}) u_{\mu 0 s}, \\
& v_{\mu p s}=B(\mu, \vec{p}) v_{\mu 0 s},
\end{aligned}
$$

where the boost matrix in the spinor representation,

$$
B(\mu, \vec{p})=\frac{1}{\sqrt{2 \mu\left(E_{\mu p}+\mu\right)}}(\not p \beta+\mu),
$$

acts on the spinors that correspond to fermions at rest. In the representation of $\gamma$-matrices used in Ref. 9], see Eq. (A2) in Appendix A the spinors at rest are the ones given in Eqs. (A8) and (A9) after multiplication by $\sqrt{2 \mu}$.

The quantum field $\hat{\psi}$ is obtained from $\psi$ by replacing the Fourier coefficients $b$ and $d$ with operators. The nonzero anti-commutation relations the resulting operators satisfy read

$$
\begin{aligned}
\left\{\hat{\psi}(\vec{x}), \hat{\psi}^{\dagger}\left(\vec{x}^{\prime}\right)\right\} & =\delta^{3}\left(\vec{x}-\vec{x}^{\prime}\right) \\
\left\{b_{\mu p s}, b_{\mu p^{\prime} s^{\prime}}^{\dagger}\right\} & =\left\{d_{\mu p s}, d_{\mu p^{\prime} s^{\prime}}^{\dagger}\right\} \\
& =2 E_{\mu p}(2 \pi)^{3} \delta^{3}\left(\vec{p}-\vec{p}^{\prime}\right) \delta_{s s^{\prime}}
\end{aligned}
$$

The quantum field $\hat{\phi}$ is obtained in a similar way keeping $\nu$ in place of $\mu$.

The quantum Hamiltonian takes the form

$$
\hat{H}=\int d^{3} x:\left[\hat{\psi}^{\dagger}(i \vec{\alpha} \vec{\partial}+\beta \mu) \hat{\psi}+\hat{\phi}^{\dagger}(i \vec{\alpha} \vec{\partial}+\beta \nu) \hat{\phi}\right]:
$$

where the symbols : denote normal ordering of the operators between them, i.e., creation operators are put to the left of the annihilation operators. Such ordering involves dropping an infinite additive numerical constant of dimension energy from the Hamiltonian.

To avoid the infinity, one would have to limit the range of momentum in the Fourier expansion of the quantum fields and the size of space volume in which the theory is being constructed. On the other hand, a numerical constant does not contribute to the resulting quantum mechanics and can be ignored. This is justified by saying (e.g., see Ref [10], p. 297) that the resulting quantum Hamiltonian has the structure

$$
\begin{aligned}
\hat{H}_{0} & =\sum_{\mu p s} E_{\mu p}\left(b_{\mu p s}^{\dagger} b_{\mu p s}+d_{\mu p s}^{\dagger} d_{\mu p s}\right) \\
& +\sum_{\nu p s} E_{\nu p}\left(b_{\nu p s}^{\dagger} b_{\nu p s}+d_{\nu p s}^{\dagger} d_{\nu p s}\right)
\end{aligned}
$$

which is physically right for counting energy of free fermions. The subscript 0 is used to indicate that there is no interaction.

All the relations given above are commonly known. They are given here for the purpose of observing that the construction of quantum fields in the IF of dynamics relies on the representation of boosts for fermions that is valid only if they are free. The issue is that in a theory with interactions the complete boost operators depend on the interactions. The boosts do not belong to the little group [4] associated with a time-like four-vector $n$ that defines the canonical quantization hyperplane in space-time through condition $n x=x^{0}=0$, where $x$ denotes the co-ordinates of points in space-time in the frame of reference of an observer who carries out the quantization procedure and whose world-line lies along $n$. The general feature of boosts depending on interaction is also exhibited in the case of the mass mixing interaction to be discussed below. Not only the mass parameters must be chosen properly in the IF quantization of fields but also the quantum creation and annihilation operators need proper definitions. Such definitions are necessary in order to avoid the IF Dirac vacuum problem [3] described in Sec. IIB below. In general, however, one does not know what mass parameters and operators to assign to fermions in the IF construction of a quantum field theory in the presence of interactions, especially in the case of strong interactions to which one cannot apply any perturbative procedure that starts from the free particle approximation. The ultimate difficulty with the free fermion mass assignment is encountered in the case of confined quarks. It is hence helpful to keep in 
mind while following further discussion of the theory of fermions with mass mixing in the IF of dynamics that the FF construction of the theory is different and does not require any assignment of masses to fermions in the definition of quantum field operators on the front where the initial conditions are specified.

\section{B. IF mass mixing and the vacuum}

The Lagrangian density including the mass mixing interaction is defined by writing

$$
\mathcal{L}=\bar{\psi}(i \not \partial-\mu) \psi+\bar{\phi}(i \not \partial-\nu) \phi-m(\bar{\psi} \phi+\bar{\phi} \psi)
$$

The corresponding quantum Hamiltonian of canonical IF quantization procedure (e.g., see Refs. [9 11]) is

$$
\hat{H}=\hat{H}_{0}+\hat{H}_{I}
$$

where $\hat{H}_{0}$ is given in Eq. (16) and the interaction term reads

$$
\hat{H}_{I}=m \int d^{3} x:\left(\hat{\psi}^{\dagger} \gamma^{0} \hat{\phi}+\hat{\phi}^{\dagger} \gamma^{0} \hat{\psi}\right):
$$

Using the Fourier expansions for the quantum fields described in previous section, integrating over space and performing normal ordering, one obtains

$$
\begin{aligned}
\hat{H}_{I} & =m \sum_{\mu p s} \sum_{s^{\prime}} \frac{1}{2 E_{\nu p}}\left[\bar{u}_{\mu p s} u_{\nu p s^{\prime}} b_{\mu p s}^{\dagger} b_{\nu p s^{\prime}}\right. \\
& +\bar{u}_{\mu p s} v_{\nu-p s^{\prime}} b_{\mu p s}^{\dagger} d_{\nu-p s^{\prime}}^{\dagger}+\bar{v}_{\mu p s} u_{\nu-p s^{\prime}} d_{\mu p s} b_{\nu-p s^{\prime}} \\
& \left.-\bar{v}_{\mu p s} v_{\nu p s^{\prime}} d_{\nu p s^{\prime}}^{\dagger} d_{\mu p s}\right]+(\mu \leftrightarrow \nu) .
\end{aligned}
$$

The Hamiltonian $\hat{H}$ can be considered an operator in the Fock space whose basis states are created from the bare vacuum state $|0\rangle$ by products of creation operators. The state $|0\rangle$ is defined by the condition that it is annihilated by all annihilation operators in the theory.

Unfortunately, the interaction Hamiltonian $\hat{H}_{I}$ is able to copiously create fermion-anti-fermion pairs from the bare vacuum state $|0\rangle$ no matter how small the mass mixing parameter $m$ is. Such creation leads to the divergences that were considered severe enough to question the existence of the Schrödinger picture in QED [3]. Indeed, the vacuum problem in the mass mixing model is an elementary example of the general vacuum problem in relativistic quantum field theory with interactions. The general vacuum problem has a long history of attempts to solve it, motivated by its basic significance in physics. The literature concerning the problem is very rich. The list of Refs. [3, 8, 12 28] amply illustrates this statement. Despite that the list is greatly incomplete and partly biased by the stress on works that concern differences between formulations of the vacuum problem in the IF and FF of dynamics, the quoted works are indicative of the development of ideas concerning the vacuum problem over recent half of a century.

Our further discussion is limited to the simple mass mixing model. We proceed to an explanation of the divergences that appear in its vacuum problem.

Consider the pair-creation term in $\hat{H}_{I}$ of Eq. (20),

$$
\hat{h}=m \sum_{\mu p s} \sum_{s^{\prime}} \frac{1}{2 E_{\nu p}} \bar{u}_{\mu p s} v_{\nu-p s^{\prime}} b_{\mu p s}^{\dagger} d_{\nu-p s^{\prime}}^{\dagger}
$$

This term is analogous to the model Hamiltonian term of Eq. (9) in Ref. [3]. The term $\hat{h}$ contains the spinor product

$$
\begin{aligned}
\bar{u}_{\mu p s} v_{\nu-p s^{\prime}} & =\left(\sqrt{\frac{E_{\nu p}+\nu}{E_{\mu p}+\mu}}+\sqrt{\frac{E_{\mu p}+\mu}{E_{\nu p}+\nu}}\right) \\
& \times \chi_{s}^{\dagger} \vec{\sigma} \vec{p} i \sigma^{2} \chi_{s^{\prime}}
\end{aligned}
$$

where the two-component spinors $\chi_{s}$ are the ones introduced in spinors of fermions at rest, $u_{\mu 0 s}$ and $v_{\mu 0 s}$ in Eqs. (10) and (11).

The eigenvalue problem for the ground state of $\hat{H}$ involves $\hat{h}$. The state obtained by acting with $\hat{h}$ on the bare vacuum,

$$
|h\rangle=\hat{h}|0\rangle
$$

differs from the bare vacuum. The question arises how to find the true ground state of the theory, if it is not $|0\rangle$.

If a part $\hat{h}$ of $\hat{H}_{I}$ produces $|h\rangle \neq 0$, the ground eigenstate of $\hat{H}$ must involve the component proportional to $|h\rangle$ once it contains a component proportional to $|0\rangle$. Then the term $\hat{h}^{\dagger}$ in the same $\hat{H}_{I}$ produces a state of an infinite norm when acting on $|h\rangle$. Further action of $\hat{h}$ and $\hat{h}^{\dagger}$ produces states with additional pairs and infinities. The ground state would have to involve some combination of all of them. Ref. [3] points out that the problem with ultra-violet divergences in all these states leads to violation of the Lorentz symmetry in a mathematically well-defined theory. In our example, the corresponding reasoning could go as follows.

Since $|h\rangle$ is an eigenstate of the three-momentum operator with eigenvalue 0 , it has, as all eigenstates of the three-momentum operator, a norm squared proportional to the volume of space, or $V=\int d^{3} x=(2 \pi)^{3} \delta^{3}(0)$. This is a general feature and it does not pose serious problems for application of a theory to the description of physical phenomena of a finite size. However, a direct evaluation yields

$$
\begin{aligned}
\langle h \mid h\rangle & =\left\langle 0\left|\hat{h}^{\dagger} \hat{h}\right| 0\right\rangle \\
& =V m^{2} \sum_{\mu p s} \sum_{s^{\prime}} \frac{1}{2 E_{\nu p}}\left|\bar{u}_{\mu p s} v_{\nu-p s^{\prime}}\right|^{2} \\
& =V m^{2} \int \frac{d^{3} p}{(2 \pi)^{3} 2 E_{\mu p}} \frac{\vec{p}^{2}}{E_{\nu p}} .
\end{aligned}
$$


This result means that the norm of $|h\rangle$ is infinite unless the number of momentum states of a single fermion in the theory is limited by some ultraviolet cutoff, say $\Lambda$, on $|\vec{p}|$. Otherwise, action of $\hat{H}_{I}$ takes states out of the Hilbert space. To obtain a mathematically acceptable theory, the Fourier expansion of the fermion fields $\hat{\psi}(\vec{x})$ and $\hat{\phi}(\vec{x})$ at $x^{0}=0$ must be cut off at some finite $\Lambda$, or regulated in some other way in the ultra-violet so that the range of momenta is effectively limited by some $\Lambda$. But every finite cutoff $\Lambda$ on particle momenta violates the Lorentz symmetry. Since this symmetry is believed to be physically valid to a great precision, the theory with a finite cutoff faces the problem of applicability in physics. In particular, the theoretical assumption that there exists a vacuum state that is invariant with respect to the Lorentz transformations is not compatible with a precisely defined theory.

The ultra-violet divergent pair creation that causes the vacuum problem also leads to divergences in other states and the Schrödinger evolution operator $\exp (-i \hat{H} t)$ cannot be understood as an operator in terms of the corresponding Taylor series acting on any state. The question then arises if a relativistic quantum theory with a mass mixing interaction can be formulated in the IF of quantum Hamiltonian dynamics. The positive answer to this question that is discussed below in Sec. IIC involves a well-known procedure that we call the IF requantization. However, although the re-quantization works for the mass mixing model, it does not tell us at all how to seek a solution of the general vacuum problem in other theories, as the half of a century of research we refer to above attests. The alternative approach that is based on the RGPEP, and can be employed to study also other theories, will be discussed later on in Sec. III]

\section{IF re-quantization}

The Lagrangian density of Eq. (17) can be written in the equivalent form

$$
\mathcal{L}=\bar{\Psi}(i \not \supset-M) \Psi,
$$

where the field $\Psi$ is a double size fermion field built from the two four-component fields $\psi$ and $\phi$,

$$
\Psi=\left[\begin{array}{l}
\psi \\
\phi
\end{array}\right]
$$

so that $\Psi$ has altogether 8 components. The mass symbol $M$ stands for the $8 \times 8$ mass matrix, formed out of four $4 \times 4$ unit matrices multiplied each by $\mu, \nu$, or $m$,

$$
M=\left[\begin{array}{cc}
\mu & m \\
m & \nu
\end{array}\right]
$$

Let the notation be arranged so that $\mu-\nu>0$. This is always possible except for the case of fermions with equal masses, i.e., $\mu=\nu$, which is special and will be commented on separately in further discussion. The eigenvalues and normalized eigenvectors of the mass matrix $M$ are

$$
\begin{aligned}
m_{1,2} & =[\mu+\nu \pm(\mu-\nu) \epsilon] / 2, \\
v_{1} & =\left[\begin{array}{r}
\cos \varphi \\
-\sin \varphi
\end{array}\right], \quad v_{2}=\left[\begin{array}{c}
\sin \varphi \\
\cos \varphi
\end{array}\right],
\end{aligned}
$$

where

$$
\begin{aligned}
\epsilon & =\sqrt{1+[2 m /(\mu-\nu)]^{2}}, \\
\varphi & =-\arctan \sqrt{\frac{\epsilon-1}{\epsilon+1}} .
\end{aligned}
$$

The double size fermion field $\Psi$ can be written in terms of two new four-component fields $\psi_{1}$ and $\psi_{2}$ using the eigenvectors of $M$,

$$
\Psi=\psi_{1} v_{1}+\psi_{2} v_{2} .
$$

The new four-component fermion fields are

$$
\begin{aligned}
& \psi_{1}=\cos \varphi \psi-\sin \varphi \phi, \\
& \psi_{2}=\sin \varphi \psi+\cos \varphi \phi .
\end{aligned}
$$

The IF re-quantization is based on expressing the classical Lagrangian density of Eq. (17) in terms of the fields $\psi_{1}$ and $\psi_{2}$. Since these fields multiply the orthogonal eigenvectors of $M$, they are multiplied in the Lagrangian by the corresponding eigenvalues $m_{1}$ and $m_{2}$ and they are not mixed by $M$. Since the Lagrangian density term with $i \not \partial$ is the same for both fields $\psi$ and $\phi$ and does not mix them, the orthogonal rotation of fields from $\psi$ and $\phi$ to $\psi_{1}$ and $\psi_{2}$ does not alter this term.

The Lagrangian density of Eq. (17) takes the form

$$
\mathcal{L}=\bar{\psi}_{1}\left(i \not \partial-m_{1}\right) \psi_{1}+\bar{\psi}_{2}\left(i \not \partial-m_{2}\right) \psi_{2} .
$$

One can now quantize the independent fields $\psi_{1}$ and $\psi_{2}$ as if they were free, because there is no interaction between them; the mass mixing is removed at the classical level of dealing with the fields. The only effect of the original mass mixing interaction is that the masses $m_{1}$ and $m_{2}$ are the eigenvalues of $M$. We call this new quantization a re-quantization because it removes the mass mixing interaction terms that caused trouble in the original quantum theory of fields $\hat{\psi}$ and $\hat{\phi}$. We have stepped back to the classical theory, introduced new field variables $\psi_{1}$ and $\psi_{2}$, and now we construct the new quantum operators $\hat{\psi}_{1}$ and $\hat{\psi}_{2}$ instead of struggling with the old ones $\hat{\psi}$ and $\hat{\phi}$.

The quantum operators $\hat{\psi}_{1}$ and $\hat{\psi}_{2}$ are obtained by imposing standard anti-commutation relations of the type indicated in Eqs. (13) and (14). Following the same steps that previously led to Eq. (16), one now obtains

$$
\begin{aligned}
\hat{H} & =\sum_{m_{1} p s} E_{m_{1} p}\left(b_{m_{1} p s}^{\dagger} b_{m_{1} p s}+d_{m_{1} p s}^{\dagger} d_{m_{1} p s}\right) \\
& +\sum_{m_{2} p s} E_{m_{2} p}\left(b_{m_{2} p s}^{\dagger} b_{m_{2} p s}+d_{m_{2} p s}^{\dagger} d_{m_{2} p s}\right)
\end{aligned}
$$


which is a quantum IF Hamiltonian for two types of free fermions with masses $m_{1}$ and $m_{2}$. The vacuum problem appears now absent because the mixing is classically included in the new mass parameters and the re-quantized theory does not produce terms of the type $b^{\dagger} d^{\dagger}$ and $d b$ any more.

The situation is similar to the one in scalar theory with mass mixing interactions discussed in Ref. [1]. Disappearance of terms such as $b^{\dagger} d^{\dagger}$ results from the choice of masses in $E_{m_{1} p}$ and $E_{m_{2} p}$. However, instead of using these energies for constructing the time derivatives of fields that play the role of canonical momenta, one constructs the corresponding spinors whose matrix elements in front of the terms such as $b^{\dagger} d^{\dagger}$ vanish.

As in the scalar case, the author does not know of any practical extension of the IF re-quantization recipe for fermion mass mixing that could be systematically applied in relativistic theories with other interactions beyond the perturbative expansion that is based on a free particle approximation with nearly precise match between the theoretical Lagrangian mass parameters and masses of physical particles. The RGPEP will be shown below to deal with the mass mixing interaction quite differently, entirely within a quantum theory of $\hat{\psi}$ and $\hat{\phi}$, i.e., without a need to define new fields $\psi_{1}$ and $\psi_{2}$ and quantizing them from scratch to define $\hat{\psi}_{1}$ and $\hat{\psi}_{2}$. This means that the RGPEP works in a way that can be systematically tried also in application to other types of interaction than just the mass mixing.

\section{FF THEORY OF MASS MIXING}

The FF of dynamics aims at description of the evolution of a system from one hyperplane of constant $x^{+}=x^{0}+x^{3}$ to another [2], with the front $x^{+}=0$ used to set up a quantum theory. We use notation $v^{ \pm}=v^{0} \pm v^{3}$ and $v^{\perp}=\left(v^{1}, v^{2}\right)$ for all four-vectors. The same convention is adopted for denoting components of all tensors. In particular, $\partial^{ \pm}=2 \partial / \partial x^{\mp}$ and $\partial^{\perp}=-\partial / \partial x^{\perp}$.

In the FF of dynamics, it is useful to consider the Lagrangian density of Eq. (17) in the form of Eq. (27). The Euler-Lagrange equations read

$$
(i \not \partial-M) \Psi=0 .
$$

Using conventions described in Appendix A, one can write these equations as

$$
i \partial^{-} \Psi_{+}+i \partial^{+} \Psi_{-}-\left(i \alpha^{\perp} \partial^{\perp}+\beta M\right)\left(\Psi_{+}+\Psi_{-}\right)=0 .
$$

Projection with $\Lambda_{+}$yields equations of motion that involve $\partial^{-}$,

$$
i \partial^{-} \Psi_{+}=\left(i \alpha^{\perp} \partial^{\perp}+\beta M\right) \Psi_{-} .
$$

Projection with $\Lambda_{-}$produces complementary constraints, i.e., equations that do not involve $\partial^{-}$,

$$
i \partial^{+} \Psi_{-}=\left(i \alpha^{\perp} \partial^{\perp}+\beta M\right) \Psi_{+} .
$$

In deriving the corresponding FF Hamiltonian, one can take advantage of Refs. [29, 30] and obtain

$$
P^{-}=\frac{1}{2} \int d x^{-} d^{2} x^{\perp} \mathcal{T}^{+-},
$$

with the energy-momentum density component

$$
\begin{aligned}
\frac{1}{2} \mathcal{T}^{+-} & =\Psi_{+}^{\dagger} i \partial^{-} \Psi_{+} \\
& =\Psi_{+}^{\dagger}\left(i \alpha^{\perp} \partial^{\perp}+\beta M\right) \frac{1}{i \partial^{+}}\left(i \alpha^{\perp} \partial^{\perp}+\beta M\right) \Psi_{+}
\end{aligned}
$$

The density involves the non-local inverse of the differential operator,

$$
\frac{1}{i \partial^{+}} f\left(x^{-}, x^{\perp}\right)=\frac{1}{2}\left(\int_{-\infty}^{x^{-}}-\int_{x^{-}}^{+\infty}\right) d y^{-} f\left(y^{-}, x^{\perp}\right) .
$$

For finite and non-zero momentum arguments of the Fourier transform $\hat{f}\left(p^{+}, p^{\perp}\right)$ of the function $f\left(x^{-}, x^{\perp}\right)$ that vanishes at the FF "spatial" infinity, this operation means simply dividing by $p^{+}$. It will be shown below that the RGPEP equations in the mass mixing model are completely independent of the momentum variables $p^{+}$and $p^{\perp}$. Therefore, one does not have to deal here with subtle aspects of modes with $p^{+}=0$.

Having accepted the inverse of $i \partial^{+}$as a division of the Fourier components by their $p^{+}$, one has

$$
P^{-}=\int d x^{-} d^{2} x^{\perp} \Psi_{+}^{\dagger} \frac{-\partial^{\perp 2}+M^{2}}{i \partial^{+}} \Psi_{+},
$$

where

$$
M^{2}=\left[\begin{array}{cc}
\mu^{2}+m^{2} & m(\mu+\nu) \\
m(\mu+\nu) & \nu^{2}+m^{2}
\end{array}\right] .
$$

The next step is to define the corresponding quantum theory.

\section{A. FF quantization}

The quantum Hamiltonian $\hat{P}^{-}$defined by

$$
\hat{P}^{-}=\int d x^{-} d^{2} x^{\perp}: \hat{\Psi}_{+}^{\dagger} \frac{-\partial^{\perp 2}+M^{2}}{i \partial^{+}} \hat{\Psi}_{+}:,
$$

can be obtained by using the representation of the $\gamma$ matrices defined in Appendix $\mathrm{A}$ and taking advantage of the results for spinors and quantization of a fermion field in Appendix B.

In analogy to Eq. (B26), one can write classical fields at $x^{+}=0$ in the form

$$
\begin{aligned}
& \psi(x)=\left[\begin{array}{l}
\zeta(x) \\
\xi(x)
\end{array}\right], \\
& \phi(x)=\left[\begin{array}{l}
\omega(x) \\
\rho(x)
\end{array}\right]
\end{aligned}
$$


In the representation of $\gamma$-matrices defined in Eqs. A22 and (A23), one has

$$
\psi_{+}(x)=\left[\begin{array}{c}
\zeta(x) \\
0
\end{array}\right], \quad \phi_{+}(x)=\left[\begin{array}{c}
\omega(x) \\
0
\end{array}\right],
$$

so that the double size fermion field $\Psi_{+}$is composed of the two-component fermion fields $\zeta(x)$ and $\omega(x)$ according to

$$
\Psi_{+}(x)=\left[\begin{array}{c}
\zeta(x) \\
0 \\
\omega(x) \\
0
\end{array}\right]
$$

The quantum fields $\hat{\psi}_{+}$and $\hat{\phi}_{+}$are obtained by changing the classical fields $\zeta(x)$ and $\omega(x)$ to operators according to the pattern of Eq. (B30), with

$$
\begin{aligned}
& \hat{\zeta}(x)=\sum_{p s} \sqrt{p^{+}}\left[b_{\zeta p s} e^{-i p x}-d_{\zeta p s}^{\dagger} e^{i p x} \sigma^{1}\right] \chi_{s}, \\
& \hat{\omega}(x)=\sum_{p s} \sqrt{p^{+}}\left[b_{\omega p s} e^{-i p x}-d_{\omega p s}^{\dagger} e^{i p x} \sigma^{1}\right] \chi_{s},
\end{aligned}
$$

where

$$
\sum_{p s}=\sum_{s= \pm 1} \int_{-\infty}^{+\infty} \frac{d^{2} p^{\perp}}{(2 \pi)^{2}} \int_{0}^{+\infty} \frac{d p^{+}}{2(2 \pi) p^{+}}
$$

and the operators $b_{\zeta p s}, d_{\zeta p s}, b_{\omega p s}$, and $d_{\omega p s}$, annihilate fermions and anti-fermions of two kinds, respectively. The non-zero canonical anti-commutation relations at $x^{+}=0$,

$$
\left\{\hat{\zeta}(x), \hat{\zeta}^{\dagger}\left(x^{\prime}\right)\right\}=\left\{\hat{\omega}(x), \hat{\omega}^{\dagger}\left(x^{\prime}\right)\right\}=\delta^{3}\left(x-x^{\prime}\right),
$$

correspond to

$$
\begin{aligned}
\left\{b_{\zeta p s}, b_{\zeta p^{\prime} s^{\prime}}^{\dagger}\right\} & =\left\{d_{\zeta p s}, d_{\zeta p^{\prime} s^{\prime}}^{\dagger}\right\} \\
& =2 p^{+}(2 \pi)^{3} \delta^{3}\left(p-p^{\prime}\right) \delta_{s s^{\prime}} \\
\left\{b_{\omega p s}, b_{\omega p^{\prime} s^{\prime}}^{\dagger}\right\} & =\left\{d_{\omega p s}, d_{\omega p^{\prime} s^{\prime}}^{\dagger}\right\} \\
& =2 p^{+}(2 \pi)^{3} \delta^{3}\left(p-p^{\prime}\right) \delta_{s s^{\prime}}
\end{aligned}
$$

The above operator representations of quantum fermion fields $\hat{\zeta}(x)$ and $\hat{\omega}(x)$ at $x^{+}=0$ are universal in the sense that they are independent of the fermion mass parameters.

In terms of the quantum fields $\hat{\zeta}(x)$ and $\hat{\omega}(x)$, the Hamiltonian of Eq. (49) reads

$$
\hat{P}^{-}=\hat{P}_{f}^{-}+\hat{P}_{I}^{-},
$$

where the free Hamiltonian is

$$
\begin{aligned}
\hat{P}_{f}^{-} & =\int d x^{-} d^{2} x^{\perp}:\left(\hat{\zeta}^{\dagger} \frac{-\partial^{\perp 2}+\mu^{2}}{i \partial^{+}} \hat{\zeta}\right. \\
& \left.+\hat{\omega}^{\dagger} \frac{-\partial^{\perp 2}+\nu^{2}}{i \partial^{+}} \hat{\omega}\right):
\end{aligned}
$$

and the interaction Hamiltonian is

$$
\begin{aligned}
\hat{P}_{I}^{-} & =\int d x^{-} d^{2} x^{\perp}:\left[\hat{\zeta}^{\dagger} \frac{m(\mu+\nu)}{i \partial^{+}} \hat{\omega}\right. \\
& \left.+\hat{\omega}^{\dagger} \frac{m(\mu+\nu)}{i \partial^{+}} \hat{\zeta}+\hat{\zeta}^{\dagger} \frac{m^{2}}{i \partial^{+}} \hat{\zeta}+\hat{\omega}^{\dagger} \frac{m^{2}}{i \partial^{+}} \hat{\omega}\right]: .
\end{aligned}
$$

The interaction contains terms linear in the mass mixing parameter $m$ and terms quadratic in $m$. The latter appear because of the FF constraint equations.

Evaluation of the Hamiltonian in terms of the creation and annihilation operators yields

$$
\begin{aligned}
\hat{P}^{-} & =\sum_{p s}\left[\left(p_{\mu}^{-}+\frac{m^{2}}{p^{+}}\right)\left(b_{\zeta p s}^{\dagger} b_{\zeta p s}+d_{\zeta p s}^{\dagger} d_{\zeta p s}\right)\right. \\
& +\left(p_{\nu}^{-}+\frac{m^{2}}{p^{+}}\right)\left(b_{\omega p s}^{\dagger} b_{\omega p s}+d_{\omega p s}^{\dagger} d_{\omega p s}\right) \\
& +\frac{m(\mu+\nu)}{p^{+}}\left(b_{\zeta p s}^{\dagger} b_{\omega p s}+d_{\omega p s}^{\dagger} d_{\zeta p s}\right. \\
& \left.\left.+b_{\omega p s}^{\dagger} b_{\zeta p s}+d_{\zeta p s}^{\dagger} d_{\omega p s}\right)\right],
\end{aligned}
$$

where

$$
p_{\mu}^{-}=\frac{p^{\perp 2}+\mu^{2}}{p^{+}}, \quad p_{\nu}^{-}=\frac{p^{\perp 2}+\nu^{2}}{p^{+}} .
$$

Note that the FF condition that all quanta have positive momentum $p^{+}$eliminates terms of the type $b^{\dagger} d^{\dagger}$ and $b d$. The negative sign in front of $d^{\dagger}$ in $\hat{\zeta}$ of Eq. (54) and $\hat{\omega}$ of Eq. (55) is compensated by the sign of inverse of $i \partial^{+}$ and the normal ordering of anti-fermion operators compensates the negative signs in front of $d$ in $\hat{\zeta}^{\dagger}$ and $\hat{\omega}^{\dagger}$. The vacuum problem is thus eliminated from the quantum theory. However, the mass mixing interaction is still present in the FF Hamiltonian of Eq. (63). This Hamiltonian provides the initial condition for the RGPEP.

\section{B. Application of the RGPEP}

The Hamiltonian $\hat{P}^{-}$of Eq. (63) is now considered an initial condition,

$$
\mathcal{P}_{0}^{-}=\hat{P}^{-},
$$

in the RGPEP scale evolution of $\mathcal{P}_{t}^{-}$according to the equation (see Appendix C)

$$
\mathcal{P}_{t}^{-^{\prime}}=\left[\left[\mathcal{P}_{f}^{-}, \mathcal{P}_{P t}^{-}\right], \mathcal{P}_{t}^{-}\right] .
$$

The prime denotes differentiation with respect to the scale parameter $t$ that ranges from 0 at the beginning and tends to $\infty$ at the end of the RGPEP evolution. The equation is further explained in Appendix C. Equation (66) is the same general RGPEP equation that is 
used in the case of boson mass mixing and can also be used in other quantum field theories.

Direct inspection of how Eq. (66) works in the fermion mass mixing model (see below) allows one to write a general solution for $\mathcal{P}_{t}^{-}$in the form

$$
\begin{aligned}
\mathcal{P}_{t}^{-} & =\sum_{p s}\left[A_{t p}\left(b_{\zeta p s}^{\dagger} b_{\zeta p s}+d_{\zeta p s}^{\dagger} d_{\zeta p s}\right)\right. \\
& +B_{t p}\left(b_{\omega p s}^{\dagger} b_{\omega p s}+d_{\omega p s}^{\dagger} d_{\omega p s}\right) \\
& +C_{t p}\left(b_{\zeta p s}^{\dagger} b_{\omega p s}+b_{\omega p s}^{\dagger} b_{\zeta p s}\right. \\
& \left.\left.+d_{\zeta p s}^{\dagger} d_{\omega p s}+d_{\omega p s}^{\dagger} d_{\zeta p s}\right)\right]
\end{aligned}
$$

where the spin-independent coefficients are

$$
\begin{aligned}
A_{t p} & =\frac{p^{\perp 2}+\mu_{t}^{2}}{p^{+}}, \\
B_{t p} & =\frac{p^{\perp 2}+\nu_{t}^{2}}{p^{+}}, \\
C_{t p} & =\frac{m_{t}^{2}}{p^{+}},
\end{aligned}
$$

and the initial conditions at $t=0 \mathrm{read}$

$$
\begin{aligned}
\mu_{0}^{2} & =\mu^{2}+m^{2}, \\
\nu_{0}^{2} & =\nu^{2}+m^{2}, \\
m_{0}^{2} & =m(\mu+\nu) .
\end{aligned}
$$

Note that the negative initial mixing term coefficient $m$ implies a negative initial value of $m_{t}^{2}$, which means that the notation $m_{t}^{2}$ is merely a formal indication that its dimension is mass squared but the value can be negative.

\section{Boost invariance}

We explain how the design of the RGPEP leads to boost invariant evolution equations for mass parameters alone, which happens because the RGPEP preserves all kinematical symmetries of the $\mathrm{FF}$ and the mass mixing interactions in $\mathcal{P}_{t}^{-}$are sufficiently simple. The formal features described below are shown in Sec. IIID to lead to the Lorentz symmetry in the spectrum of solutions in the model fermion theory.

According to the general RGPEP rules described in Appendix [C] the operators $\mathcal{P}_{f}^{-}$and $\mathcal{P}_{P t}^{-}$in Eq. (66) are

$$
\begin{aligned}
\mathcal{P}_{f}^{-} & =\sum_{p s}\left[p_{\mu}^{-}\left(b_{\zeta p s}^{\dagger} b_{\zeta p s}+d_{\zeta p s}^{\dagger} d_{\zeta p s}\right)\right. \\
& \left.+p_{\nu}^{-}\left(b_{\omega p s}^{\dagger} b_{\omega p s}+d_{\omega p s}^{\dagger} d_{\omega p s}\right)\right] \\
\mathcal{P}_{P t}^{-} & =\sum_{p s} p^{+2}\left[A_{t p}\left(b_{\zeta p s}^{\dagger} b_{\zeta p s}+d_{\zeta p s}^{\dagger} d_{\zeta p s}\right)\right. \\
& +B_{t p}\left(b_{\omega p s}^{\dagger} b_{\omega p s}+d_{\omega p s}^{\dagger} d_{\omega p s}\right) \\
& +C_{t p}\left(b_{\zeta p s}^{\dagger} b_{\omega p s}+b_{\omega p s}^{\dagger} b_{\zeta p s}\right. \\
& \left.\left.+d_{\zeta p s}^{\dagger} d_{\omega p s}+d_{\omega p s}^{\dagger} d_{\zeta p s}\right)\right]
\end{aligned}
$$

The resulting RGPEP generator is

$$
\begin{aligned}
{\left[\mathcal{P}_{f}^{-}, \mathcal{P}_{P t}^{-}\right] } & =\sum_{p s} C_{t p} p^{+2}\left(p_{\mu}^{-}-p_{\nu}^{-}\right)\left(b_{\zeta p s}^{\dagger} b_{\omega p s}\right. \\
& \left.-b_{\omega p s}^{\dagger} b_{\zeta p s}+d_{\zeta p s}^{\dagger} d_{\omega p s}-d_{\omega p s}^{\dagger} d_{\zeta p s}\right)
\end{aligned}
$$

Consequently, Eq. (66) reads

$$
\begin{aligned}
\mathcal{P}_{t}^{-\prime} & =\sum_{p s}\left[A_{t p}^{\prime}\left(b_{\zeta p s}^{\dagger} b_{\zeta p s}+d_{\zeta p s}^{\dagger} d_{\zeta p s}\right)\right. \\
& +B_{t p}^{\prime}\left(b_{\omega p s}^{\dagger} b_{\omega p s}+d_{\omega p s}^{\dagger} d_{\omega p s}\right) \\
& +C_{t p}^{\prime}\left(b_{\zeta p s}^{\dagger} b_{\omega p s}+b_{\omega p s}^{\dagger} b_{\zeta p s}\right. \\
& \left.\left.+d_{\zeta p s}^{\dagger} d_{\omega p s}+d_{\omega p s}^{\dagger} d_{\zeta p s}\right)\right] \\
& =-\sum_{p s} C_{t p} p^{+2}\left(p_{\mu}^{-}-p_{\nu}^{-}\right)\left(A_{t p}-B_{t p}\right) \\
& \times\left(b_{\zeta p s}^{\dagger} b_{\omega p s}+d_{\omega p s}^{\dagger} d_{\zeta p s}+b_{\omega p s}^{\dagger} b_{\zeta p s}+d_{\zeta p s}^{\dagger} d_{\omega p s}\right) \\
& +\sum_{p s} 2 C_{t p}^{2} p^{+2}\left(p_{\mu}^{-}-p_{\nu}^{-}\right) \\
& \times\left[b_{\zeta p s}^{\dagger} b_{\zeta p s}+d_{\zeta p s}^{\dagger} d_{\zeta p s}-b_{\omega p s}^{\dagger} b_{\omega p s}-d_{\omega p s}^{\dagger} d_{\omega p s}\right]
\end{aligned}
$$

Equating coefficients in front of the same operators on both sides of the last equation, one arrives at an infinite set of equations; 6 for every momentum mode $p$, i.e., 3 equations for every choice of $p$ and spin $s$. Namely,

$$
\begin{aligned}
A_{t p}^{\prime} & =2 p^{+2}\left(p_{\mu}^{-}-p_{\nu}^{-}\right) C_{t p}^{2} \\
B_{t p}^{\prime} & =-2 p^{+2}\left(p_{\mu}^{-}-p_{\nu}^{-}\right) C_{t p}^{2} \\
C_{t p}^{\prime} & =-p^{+2}\left(p_{\mu}^{-}-p_{\nu}^{-}\right)\left(A_{t p}-B_{t p}\right) C_{t p} .
\end{aligned}
$$

The equations are independent of spin. Moreover, they can be written using Eqs. (68), (69) and (70) as

$$
\begin{aligned}
\left(\frac{p^{\perp 2}+\mu_{t}^{2}}{p^{+}}\right)^{\prime} & =2 p^{+2}\left(\frac{p^{\perp 2}+\mu^{2}}{p^{+}}-\frac{p^{\perp 2}+\nu^{2}}{p^{+}}\right) \\
& \times\left(\frac{m_{t}^{2}}{p^{+}}\right)^{2}, \\
\left(\frac{p^{\perp 2}+\nu_{t}^{2}}{p^{+}}\right)^{\prime} & =-2 p^{+2}\left(\frac{p^{\perp 2}+\mu^{2}}{p^{+}}-\frac{p^{\perp 2}+\nu^{2}}{p^{+}}\right) \\
& \times\left(\frac{m_{t}^{2}}{p^{+}}\right)^{2}, \\
\left(\frac{m_{t}^{2}}{p^{+}}\right)^{\prime}= & -p^{+2}\left(\frac{p^{\perp 2}+\mu^{2}}{p^{+}}-\frac{p^{\perp 2}+\nu^{2}}{p^{+}}\right) \\
& \times\left(\frac{p^{\perp 2}+\mu_{t}^{2}}{p^{+}}-\frac{p^{\perp 2}+\nu_{t}^{2}}{p^{+}}\right)\left(\frac{m_{t}^{2}}{p^{+}}\right) .
\end{aligned}
$$


It is visible that the momentum variables $p^{+}$and $p^{\perp}$ drop out from the infinite set of equations and every momentum mode $p$ in the FF Fourier expansion of quantum fields evolves independently of its spin and only to the extent that the mass parameters evolve. These parameters evolve according to the set of just 3 equations, which is the same for all momentum modes and spins, see Eqs. (85), (86) and (87) below.

\section{Evolution of effective mass parameters}

The RGPEP equations for the mass parameters are

$$
\begin{aligned}
\left(\mu_{t}^{2}\right)^{\prime} & =2\left(\mu^{2}-\nu^{2}\right)\left(m_{t}^{2}\right)^{2} \\
\left(\nu_{t}^{2}\right)^{\prime} & =-2\left(\mu^{2}-\nu^{2}\right)\left(m_{t}^{2}\right)^{2} \\
\left(m_{t}^{2}\right)^{\prime} & =-\left(\mu^{2}-\nu^{2}\right)\left(\mu_{t}^{2}-\nu_{t}^{2}\right) m_{t}^{2} .
\end{aligned}
$$

These equations for fermions are identical to Eqs. (53), (54), and (55) for bosons in Ref. [1], respectively. They would have the same solutions for the same initial conditions. However, the initial conditions for the fermion mass mixing Hamiltonian are different from the initial conditions for scalar boson mass mixing Hamiltonian. The difference originates in the constraints that fermions obey and scalar bosons do not. Solutions for fermions are discussed in Sec. IIIC

Note that the replacement of the constant free Hamiltonian $\mathcal{H}_{f}$ in Eq. (66) by the part of the Hamiltonian that contains the operators $b_{\zeta}^{\dagger} b_{\zeta}+d_{\zeta}^{\dagger} d_{\zeta}$ and $b_{\omega}^{\dagger} b_{\omega}+d_{\omega}^{\dagger} d_{\omega}$, which means a change in the RGPEP generator mentioned below Eq. (C8) in Appendix C] yields a slightly different set of equations,

$$
\begin{aligned}
\left(\mu_{t}^{2}\right)^{\prime} & =2\left(\mu_{t}^{2}-\nu_{t}^{2}\right)\left(m_{t}^{2}\right)^{2}, \\
\left(\nu_{t}^{2}\right)^{\prime} & =-2\left(\mu_{t}^{2}-\nu_{t}^{2}\right)\left(m_{t}^{2}\right)^{2}, \\
\left(m_{t}^{2}\right)^{\prime} & =-\left(\mu_{t}^{2}-\nu_{t}^{2}\right)^{2} m_{t}^{2} .
\end{aligned}
$$

This set of 3 equations matches the matrix Eq. (A1) in [1] that resembles Wegner's equation [31 33] for $2 \times 2$ Hamiltonian matrices. Again, since these equations are the same as for bosons, the only difference between the fermion and boson solutions comes from the initial conditions that reflect the presence of constraints for fermions. Solutions to Eqs. (88), (89) and (90) lead to the same results for $t \rightarrow \infty$ as solutions to Eqs. (85), (86) and (87) discussed below.

\section{Solutions to the RGPEP equations}

Solutions to Eqs. (85), (86), and (87) are derived in Appendix D. They differ from the solutions for bosons with mass mixing [1] due to the change of initial conditions for mass terms,

$$
\left[\begin{array}{cc}
\mu^{2} & m^{2} \\
m^{2} & \nu^{2}
\end{array}\right] \rightarrow\left[\begin{array}{cc}
\mu^{2}+m^{2} & m(\mu+\nu) \\
m(\mu+\nu) & \nu^{2}+m^{2}
\end{array}\right]
$$

The fermion initial conditions are the square of matrix $M$ in Eq. (29). See also Eqs. (47) and (48) to recall how $M^{2}$ emerges due to constraints. As a consequence, the parameter $\epsilon$ defined by Eq. (32) in the fermion case replaces the boson parameter $\epsilon=\left\{1+\left[2 m^{2} /\left(\mu^{2}-\nu^{2}\right)\right]^{2}\right\}^{1 / 2}$ when $m^{2}$ is replaced by $m(\mu+\nu)$ and the ratio $2 m^{2} /\left(\mu^{2}-\nu^{2}\right)$ becomes $2 m /(\mu-\nu)$.

Solutions of the RGPEP equations yield the diagonal form of $M^{2}$ when $t \rightarrow \infty$ for $\mu>\nu$, see Appendix D. The case $\mu=\nu$ is commented on below. The eigenvalues of $M^{2}$ are $m_{1}^{2}$ and $m_{2}^{2}$, where $m_{1}$ and $m_{2}$ are the eigenvalues of $M$ given in Eq. (30). Since $M$ is hermitian, its eigenvalues are real. This means that the eigenvalues of $M^{2}$ cannot be negative no matter how strong the mixing parameter $m$ is. This feature distinguishes the mass mixing for fermions from mass mixing for bosons. The difference is further discussed below.

The eigenvectors of $M^{2}$ are the same as eigenvectors of $M$. Therefore, the angle of rotation $\varphi$ that appears in the eigenvectors $v_{1}$ and $v_{2}$ in Eq. (31) is reproduced in the RGPEP when $t \rightarrow \infty$. This is how the RGPEP solves the mass mixing theory without any need for requantization.

We also observe that solutions to the RGPEP Eqs. (88), (89), and (90) that are obtained using the generator with running effective masses, produce the same values of masses in the limit $t \rightarrow \infty$. For finite values of $t$, the corresponding angle of rotation $\varphi_{t}$ (see Appendix (D) is different but otherwise there is no difference in comparison to solutions to Eqs. (85), (86), and (87) with constant masses in the generator.

In solving the RGPEP equations, as described in Appendix D, a convenient variable in place of $t$ is $u=\delta \mu^{4} t$, where $\delta \mu^{2}=\mu^{2}-\nu^{2}>0$. If $\mu^{2}=\nu^{2}$, the mass parameters do not evolve with $t$, irrespective of the initial value of mass-mixing parameter $m$. In this special case, one can introduce an auxiliary difference between $\mu$ and $\nu$ and one can seek solutions in the limit of the auxiliary difference going to 0 , as had already been suggested in Ref. 1]. For example, such artificial splitting of degenerated fermion masses would have to be introduced in the case of local theories with massless fermions, including theories with chiral symmetry. A prominent example of the FF quanta for which a small deviation from mass degeneracy is involved in defining the parameter $u$ are neutrinos [34].

\section{Spectrum of the theory}

The initial Hamiltonian, $\hat{P}^{-}$in Eq. (63), is transformed as a result of the RGPEP to

$$
\hat{P}^{-}=\mathcal{U}_{t} \mathcal{P}_{t}^{-} \mathcal{U}_{t}^{\dagger}
$$


where $\mathcal{P}_{t}^{-}$is given in Eq. (67) and $\mathcal{U}_{t}$ is taken from Eq. (D24). Thus,

$$
\begin{aligned}
\hat{P}^{-} & =\sum_{p s}\left[A_{t p}\left(b_{t \zeta p s}^{\dagger} b_{t \zeta p s}+d_{t \zeta p s}^{\dagger} d_{t \zeta p s}\right)\right. \\
& +B_{t p}\left(b_{t \omega p s}^{\dagger} b_{t \omega p s}+d_{t \omega p s}^{\dagger} d_{t \omega p s}\right) \\
& +C_{t p}\left(b_{t \zeta p s}^{\dagger} b_{t \omega p s}+b_{t \omega p s}^{\dagger} b_{t \zeta p s}\right. \\
& \left.\left.+d_{t \zeta p s}^{\dagger} d_{t \omega p s}+d_{t \omega p s}^{\dagger} d_{t \zeta p s}\right)\right]
\end{aligned}
$$

where the $t$-dependent annihilation operators are defined by Eqs. (D41), (D42), (D43), and (D44). The corresponding creation operators are defined through hermitian conjugation. The coefficients $A_{t p}, B_{t p}, C_{t p}$ are defined in Eqs. (68), (69) and (70), and the $t$-dependent mass parameters in them are given in Eqs. (D13), (D14), (D15), (D16). The RGPEP secures that the Hamiltonian $\hat{P}^{-}$as an operator does not depend on $t$ while the creation and annihilation operators and coefficients of their products in $\hat{P}^{-}$do depend on $t$, in such a way that in the limit of $t \rightarrow \infty$ the mass mixing term disappears, $\lim _{t \rightarrow \infty} C_{t p}=0$.

The eigenvalues and eigenstates of $\hat{P}^{-}$do not depend on $t$. One can construct the eigenstates using creation operators corresponding to any value of $t$ one chooses. Having chosen operators for some selected value of $t$, one can apply them to the bare vacuum state $|0\rangle$ and create a basis in the FF Fock space. The bare vacuum does not depend on $t$ (it is annihilated by all annihilation operators, irrespective of the value of $t$ ). If one chooses certain $t$ for creation and annihilation operators and construction of the Fock-space basis, the easiest Hamiltonian to work with is the one expressed in terms of the same operators.

In principle, one can also work with different operators for constructing states and Hamiltonians. This option involves potentially complex formulae that include logarithms and other functions of the ratios of corresponding scales in complex theories. For example, such setup is useful in the description of form factors and structure functions of hadrons because the external probes may distinguish a considerably different scale from the one that is most convenient for solving the hadron mass eigenvalue problem. The scale evolution of the parton distributions appears in the transformation matrix between the effective quanta used in the eigenvalue equation and the effective quanta corresponding the external probe scale [26].

The wave functions of eigenstates in the basis constructed at some $t$ depend on $t$. In general, the larger $t$ the more limited the spread of wave functions in total invariant masses of constituent states around the eigenvalue mass squared. In the fermion mass mixing case, the wave functions are simple to describe because one knows them exactly.

\section{The limit of $t \rightarrow \infty$}

The simplest choice of $t$ to work with is $t \rightarrow \infty$, since in this case there is no mass mixing, $C_{\infty p}=m_{\infty}^{2} / p^{+}=0$. Thus, the effective theory with $t=\infty$ is a theory of free fermions with masses $m_{1}$ and $m_{2}$, with a correspondingly simple spectrum. Namely, in the limit of $t \rightarrow \infty$,

$$
\begin{aligned}
\hat{P}^{-} & =\sum_{p s}\left[\frac{p^{\perp 2}+m_{1}^{2}}{p^{+}}\left(b_{\infty \zeta p s}^{\dagger} b_{\infty \zeta p s}+d_{\infty \zeta p s}^{\dagger} d_{\infty \zeta p s}\right)\right. \\
& \left.+\frac{p^{\perp 2}+m_{2}^{2}}{p^{+}}\left(b_{\infty \omega p s}^{\dagger} b_{\infty \omega p s}+d_{\infty \omega p s}^{\dagger} d_{\infty \omega p s}\right)\right],(94)
\end{aligned}
$$

where the operators with subscript $\infty$ are given in Eqs. (D41), (D42), (D43), and (D44) with $t=\infty$, i.e., with the angle $\varphi_{\infty}$ given by Eq. (D52), matching the angle $\varphi$ found in Eq. (33) as an ingredient of the IF requantization procedure in Sec. IIC.

$$
\varphi_{\infty}=\varphi=-\arctan \sqrt{\frac{\epsilon-1}{\epsilon+1}} .
$$

Hence, the eigenvalues of the Hamiltonian $\hat{P}^{-}$in Eq. (94) are free $\mathrm{FF}$ energies of $n_{\infty 1}$ fermions and anti-fermions of mass $m_{1}$ and $n_{\infty 2}$ fermions and anti-fermions of mass $m_{2}$, each with some momentum components $p^{+}$and $p^{\perp}$ and spin $z$-axis projection $s$, no more than 1 particle in any state with the same momentum and spin (i.e., in agreement with the Pauli exclusion principle for effective fermions),

$$
\begin{array}{r}
P_{\left\{\left(p_{1 i}, s_{1 i}\right), i=1, \ldots, n_{\infty 1}\right\},\left\{\left(p_{2 j}, s_{2 j}\right), j=1, \ldots, n_{\infty 2}\right\}} \\
=\sum_{i=1}^{n_{\infty 1}} \frac{p_{1 i}^{\perp}{ }^{2}+m_{1}^{2}}{p_{1 i}^{+}}+\sum_{j=1}^{n_{\infty} 2} \frac{p_{2 j}^{\perp}+m_{2}^{2}}{p_{2 j}^{+}} .
\end{array}
$$

The spectrum is degenerate. The eigenstates can be closely identified because the RGPEP provides expressions for the corresponding creation operators. A complete set of eigenstates (not normalized) is defined by writing

$$
\begin{aligned}
\left\{n_{\infty 1}\right\} & =\left\{\left(p_{1 i}, s_{1 i}\right), i=1, \ldots, n_{\infty 1}\right\} \\
\left\{n_{\infty 2}\right\} & =\left\{\left(p_{2 j}, s_{2 j}\right), j=1, \ldots, n_{\infty 2}\right\} \\
\left|\left\{n_{\infty 1}\right\},\left\{n_{\infty 2}\right\}\right\rangle & =\prod_{i=1}^{n_{\infty} 1}\left(b_{\infty \zeta p_{1 i} s_{1 i}}^{\dagger} \text { or } d_{\infty \zeta p_{1 i} s_{1 i}}^{\dagger}\right) \\
& \times \prod_{j=1}^{n_{\infty 2}}\left(b_{\infty \omega p_{2 j} s_{2 j}}^{\dagger} \text { or } d_{\infty \omega p_{2 i} s_{2 i}}^{\dagger}\right)|0\rangle
\end{aligned}
$$

where $|0\rangle$ denotes the bare vacuum state. $|0\rangle$ is annihilated by all annihilation operators for all values of $t$ and it can be treated as one and the same state for all values of $t$.

The eigenstates in Eq. (99) can also be written as combinations of states created from the same vacuum state 
by products of the creation operators in the FF Fourier expansions of the fields $\hat{\zeta}$ and $\hat{\omega}$ in Eqs. (54) and (55) at $t=0$. For states with a large number of fermions, a simple eigenstate made of the effective particles with $t=\infty$, i.e., physical fermions, is a complex mixture of many states made of bare particles corresponding to $t=0$.

\section{2. $\quad$ Strong mass mixing}

The mass mixing interaction with $|m|>\sqrt{\mu \nu}$ causes the smaller one of two eigenvalues of mass matrix $M, m_{2}$ in Eq. (30), to be negative. The RGPEP equations imply that $m_{2}^{2}$ is the square of mass of a physical fermion. The sign of $m_{2}$ remains undetermined by the equations of RGPEP because, due to the FF constraints, the Hamiltonian depends only on $M^{2}$. The eigenvectors of $M^{2}$ are the same as eigenvectors of $M$ but there is a difference between evolving $M^{2}$ in the RGPEP and diagonalizing $M$ in the IF re-quantization.

For $\epsilon>1$, which is obtained assuming $\mu>\nu$ and $m \neq 0$ in Eq. (17) or, equivalently, Eq. (27), the smaller one of two diagonal elements of evolving mass matrix squared, denoted by $\nu_{t}^{2}$ in Eq. (69) and Appendix D, decreases monotonically to its lowest value of $m_{2}^{2} \geq 0$, never reaching 0 if the eigenvalue $m_{2} \neq 0$. Thus, the RGPEP approaches physical solution for $\nu_{\infty}^{2}$ when $t \rightarrow \infty$ without ever referring to the sign of $\nu_{t}$. The question of a strong mass mixing with $|m|>\sqrt{\mu \nu}$ is: Where is the information about the sign of the negative eigenvalue $m_{2}$ of the mass matrix $M$ stored?

Using Eq. (C1) for the unconstrained fermion fields (and variable $t=s^{4}$ instead of $s$ ), one can write

$$
\hat{\Psi}_{t+}=\mathcal{U}_{t} \hat{\Psi}_{0+} \mathcal{U}_{t}^{\dagger}
$$

This relation describes the basic transformation of quantum degrees of freedom. The complementary field components $\Psi_{t-}$ can be obtained from the constraints they obey. The constraints involve interactions, which in our case are just the mass mixing terms that are linear, not quadratic in the mass parameters. This is where the sign of $m_{2 t}$ appears. Namely,

$$
\hat{\Psi}_{t-}=\frac{1}{i \partial^{+}}\left(i \alpha^{\perp} \partial^{\perp}+\beta M_{t}\right) \hat{\Psi}_{t+},
$$

where the matrix $M_{t}$ is a root of $M_{t}^{2}$. If one writes

$$
M_{t}^{2}=\left[\begin{array}{ll}
m_{1 t} & m_{I t} \\
m_{I t} & m_{2 t}
\end{array}\right]^{2}=\left[\begin{array}{cc}
\mu_{t}^{2} & m_{t}^{2} \\
m_{t}^{2} & \nu_{t}^{2}
\end{array}\right]
$$

the smooth solution for $M_{t}$ that satisfies the initial conditions is given by

$$
\begin{aligned}
m_{1 t} & =\frac{1}{2}\left(\mu+\nu+\frac{\delta \mu_{t}^{2}}{\mu+\nu}\right), \\
m_{2 t} & =\frac{1}{2}\left(\mu+\nu-\frac{\delta \mu_{t}^{2}}{\mu+\nu}\right), \\
m_{I t} & =\frac{m_{t}^{2}}{\mu+\nu}
\end{aligned}
$$

where $\delta \mu_{t}^{2}$ and $m_{t}^{2}$ are given in Eqs. (D15) and (D16). The mass mixing term has the same sign that $m_{t}^{2}$ has, $m_{1 t}$ monotonically increases from the positive initial fermion mass $\mu$ to the eigenvalue mass $m_{1}$, and $m_{2 t}$ monotonically decreases from the positive initial fermion mass $\nu$ to the eigenvalue mass $m_{2}$, which may become negative even if $m^{2}+\nu_{t}^{2}$ decreases monotonically from $\nu^{2}$ to $m_{2}^{2}$ and thus never approaches 0 . Thus, one obtains

$$
\begin{aligned}
\hat{\Psi}_{t} & =\hat{\Psi}_{t+}+\hat{\Psi}_{t-} \\
& =\left[\begin{array}{c}
\hat{\psi}_{1 t}+\beta m_{I t} \hat{\psi}_{2 t+} \\
\hat{\psi}_{2 t}+\beta m_{I t} \hat{\psi}_{1 t+}
\end{array}\right],
\end{aligned}
$$

where the quantum fields

$$
\begin{aligned}
& \hat{\psi}_{1 t}=\left[\begin{array}{c}
\hat{\zeta}_{t} \\
\left(i \partial^{+}\right)^{-1}\left(\sigma^{2} \partial^{1}-\sigma^{1} \partial^{2}+m_{1 t}\right) \hat{\zeta}_{t}
\end{array}\right], \\
& \hat{\psi}_{2 t}=\left[\left(i \partial^{+}\right)^{-1}\left(\sigma^{2} \partial^{1}-\sigma^{1} \partial^{2}+m_{2 t}\right) \hat{\omega}_{t}\right] \text {, }
\end{aligned}
$$

both have the FF Fourier expansions at $x^{+}=0$ of the form

$$
\begin{aligned}
\hat{\psi}_{l t}(x) & =\hat{\psi}_{l t+}(x)+\hat{\psi}_{l t-}(x) \\
& =\sum_{p s}\left[u_{p s}\left(m_{l t}\right) b_{t l p s} e^{-i p x}+v_{p s}\left(m_{l t}\right) d_{t l p s}^{\dagger} e^{i p x}\right],
\end{aligned}
$$

where $l=1$ refers to $\hat{\zeta}, l=2$ to $\hat{\omega}$, the spinors are

$$
u_{p s}\left(m_{l t}\right)=\frac{1}{\sqrt{\left|p^{+}\right|}}\left[\begin{array}{c}
p^{+} \\
-i \sigma^{2} p^{1}+i \sigma^{1} p^{2}+m_{l t}
\end{array}\right] \chi_{s},
$$

$$
v_{p s}\left(m_{l t}\right)=\frac{1}{\sqrt{\left|p^{+}\right|}}\left[\begin{array}{c}
-p^{+} \\
+i \sigma^{2} p^{1}-i \sigma^{1} p^{2}+m_{l t}
\end{array}\right] \chi_{-s},
$$

and the annihilation operators are defined according to Eqs. (D41), (D42), (D43), and (D44). In summary, the quantum field operators $\hat{\psi}_{1 t}$ and $\hat{\psi}_{2 t}$ handle the effective fermions of masses $m_{1 t}$ and $m_{2 t}$ that interact through the mass mixing interaction of strength $m_{I t}$.

When $m^{2}>\mu \nu$, the limit of $t \rightarrow \infty$ produces negative $m_{2 t}$ in $\hat{\Psi}_{t}$ and $m_{I t} \rightarrow 0$ in the Hamiltonian. On the other hand, the sign of the mass term with respect to the momentum dependent terms in the spinors in $\hat{\Psi}_{t}$ can be changed by making a chiral rotation. In the FF representation of $\gamma$-matrices, it is visible that chiral rotations turn the spin-up and spin-down +-components of spinor fields by opposite angles and the terms proportional to mass are turned by the angle opposite to the terms that are proportional to $p^{+}$and $p^{\perp}$. Rotation by angle $\pi / 2$ changes the sign of the mass terms with respect to the momentum-dependent terms. 
It might seem that one could make a chiral rotation of $\psi_{2 t}$ and restore the positivity of the mass term with $m_{2 t}$ as soon as $m_{2 t}$ changes sign as a function of $t$. However, as long as the mass mixing interaction term with $m_{I t}$ is present, the chiral rotation influences the interaction with $\psi_{1 t}$. Only for $t \rightarrow \infty$, when $m_{I t}$ vanishes, one can chirally rotate the field $\psi_{2 \infty}$ independently of the field $\psi_{1 \infty}$, both fields representing physical particles. The strong mass mixing thus produces physical fermions that are chirally rotated with respect to the fermions one starts from.

\section{Effective fermions}

The effective quantum field operators $\psi_{1 t}$ and $\psi_{2 t}$ are constructed in Eq. (110) in Sec. IIID2 according to a general scheme for building effective quantum field operators using the RGPEP, see Appendix C. The FF Hamiltonian does not change as a result of re-writing it in terms of the effective fermion operators. The constancy of the Hamiltonian as a whole includes the infinite additive constant dropped in the process of normal ordering. The constant does not depend on $t$ because the range of kinematical momentum variables does not depend on the interaction and is the same for all values of $t$. However, the mass mixing interaction strength $m_{I t}$ decreases when $t$ increases, becoming 0 in the limit $t \rightarrow \infty$, where the same Hamiltonian is expressed in terms of the creation and annihilation operators for physical fermions.

The Hamiltonian expressed in terms of the effective quantum fermion fields corresponding to $t$ is characterized by two features. One of them is that the masses $m_{1 t}$ and $m_{2 t}$ differ from the physical masses $m_{1}$ and $m_{2}$ for as long as $t$ is kept finite instead of being sent to $\infty$. The other feature is that the interaction term strength $m_{I t}$ is different from 0 for as long as $t$ is kept finite. These features of our simple model solution suggest that it may be appropriate also in more complex models, where exact solutions are not known, to keep due mass mixing interactions intact in an effective theory for as long as the effective theory includes any interactions that are capable of contributing to the effective mass mixing parameters. By the same token, it may be misleading to interpret a theory in terms of the degrees of freedom that correspond to a diagonalized mass matrix when the other interactions are present.

When there are more than just two different species of fermions mixed by mass terms, say $f$ different species corresponding to different "flavors" or "families," the RGPEP leads to equations for matrices of dimension $f \times f$. Such equations do not have known analytic solutions for a general choice of initial conditions but they do have exact numerical solutions that can be found using computers.

\section{CONCLUSION}

It has been demonstrated in Ref. [1] that the RGPEP provides a solution to the quantum theory of two kinds of scalar bosons that interact with each other through mass-mixing terms. The solution avoided the divergent vacuum problem of the type that was for a long time considered critical to construction of a relativistic quantum theory of particles and fields [3]. In this article, it is shown that the RGPEP also provides a solution to the FF theory of two kinds of spin- $1 / 2$ fermions that interact through mass-mixing terms, avoiding the associated fermion vacuum problem as well.

The differential RGPEP equations for effective mass parameters in the theories of bosons and fermions turn out to have identical forms. However, the same equations lead to qualitatively different solutions in the fermion and boson theories when the mass mixing interactions are strong. The reason is that the initial conditions in these theories are set in different ways. Since the RGPEP does not a priori rely on any perturbative expansion, it can tell us precisely what happens in the theories with arbitrary strength of the interactions.

The $2 \times 2$ mass matrix that appears in the scalar bosons theory as an initial condition for the RGPEP differential equations in a suitable operator basis has the form (see Ref. [1])

$$
M_{B}^{2}=\left[\begin{array}{cc}
\mu^{2} & m^{2} \\
m^{2} & \nu^{2}
\end{array}\right],
$$

while the analogous initial condition in the fermion theory has the form (see Sec. IIIC)

$$
M_{F}^{2}=\left[\begin{array}{cc}
\mu^{2}+m^{2} & (\mu+\nu) m \\
(\mu+\nu) m & \nu^{2}+m^{2}
\end{array}\right] .
$$

In both cases, $\mu$ and $\nu$ denote the bare masses of initial quanta and $m$ denotes the strength of the mass mixing interaction terms. Once these initial conditions are set, the RGPEP yields exact solutions for the creation and annihilation operators of effective particles and the corresponding masses in the effective Hamiltonians as functions of the scale parameter $t$. The parameter can take any value starting at 0 and ending at $\infty$. At the end of the RGPEP evolution, when $t \rightarrow \infty$, one obtains quantum Hamiltonians expressed in terms of the creation and annihilation operators for physical particles. The masses squared of the physical bosons and fermions are the eigenvalues of the matrices $M_{B}^{2}$ and $M_{F}^{2}$, respectively.

The key difference between $M_{B}^{2}$ and $M_{F}^{2}$ is that one of the eigenvalues of $M_{B}^{2}$ is negative when $|m|$ is larger than $\sqrt{\mu \nu}$ while $M_{F}^{2}$ does not have negative eigenvalues no matter how large is $m$. The wrong sign of the mass squared for bosons causes that the eigenvalues of $\hat{P}^{-}$ are unbounded from below. The fermion theory qualitatively differs from the boson theory because the wrong sign of mass squared never appears in fermion theory. This is a consequence of the fermion constraint equations 
that are specific to the FF of Hamiltonian dynamics and do not appear in theories of scalar bosons. These constraints produce the diagonal terms $\mathrm{m}^{2}$ that prevent the off-diagonal mass mixing terms $m(\mu+\nu)$ from inducing a negative mass squared for fermions no matter how large is $m$ in comparison to $\mu$ and $\nu$ and what its sign is. In other words, the FF theory of bosons with mass mixing interactions may collapse due to tachyon solutions when the interaction is strong while the FF theory of fermions cannot have tachyon solutions and cannot so collapse.

One can speculate about what may be found when the RGPEP is applied to theories with fermions that include interactions other than the mass mixing. The fermion mass may appear not only quadratically but also linearly in physically relevant interactions. The terms linear in masses can be considerably different from the mass mixing terms in our simple model. For example, fermion masses appear linearly in the photon-electron interaction terms in QED and in quark-gluon interaction terms in QCD. Perhaps the effective masses of lightest fermion species could change sign in the RGPEP due to the interactions if the latter have sufficient strength (considerably greater than in QED). If this happens, the interactions that are linear in the lightest fermion masses and hence sensitive to their signs could go through zero. Thus, the RGPEP could possibly unveil new features of relevant effective theories due to the associated chiral rotations. One might be even forced to limit the range of allowed strengths of interactions. On the other hand, the RGPEP solutions for the mass mixing in both scalar boson and spin-1/2 fermion theories suggest that interactions of weak strength in comparison to masses can hardly cause harm such as a collapse due to tachyon solutions.

Even if the lessons learned in the elementary models with mass mixing and no other interaction are insufficient to guess the nature of approximate solutions that the RGPEP may produce in complex theories, both the boson and fermion examples already indicate that the RGPEP is capable of helping in studies of quantum field theories. Some help is certainly needed in generating effective interactions in a FF theory of neutrino oscillations [34], including generation of the neutrino mass terms. Since the vacuum problem in the IF of dynamics is far from being understood, the RGPEP is of particular relevance as a method of study because it appears prepared to provide new information without changing the trivial nature of the FF vacuum state. This special feature may remain valid even in theories as complex as QCD, if the features typically associated with a complex vacuum in the IF of dynamics are instead associated with a potentially rich structure of the FF effective Hamiltonian operators. Such possibility had been previously suggested in Ref. [8]. If that guess is right, the challenge for the RGPEP is to produce the required counterterms and generate new interaction terms in effective theories.

\section{Appendix A: FF representation of $\gamma$-matrices}

The popular representation of $\gamma$-matrices [35] adopted in [9], is called below the IF representation. The IF representation leads to the $\mathrm{FF}$ projection matrices $\Lambda_{ \pm}=\gamma^{0} \gamma^{ \pm} / 2$ that mix all four components of the Dirac spinors. This Appendix defines the representation of $\gamma$ matrices, called below the FF representation, in which

$$
\Lambda_{+}=\left[\begin{array}{ll}
1 & 0 \\
0 & 0
\end{array}\right], \quad \Lambda_{-}=\left[\begin{array}{ll}
0 & 0 \\
0 & 1
\end{array}\right]
$$

In the $\mathrm{FF}$ representation, the unconstrained parts of the fermion fields $\psi$, i.e., $\psi_{+}=\Lambda_{+} \psi$, form the two upper components of $\psi$, and the dependent parts, i.e., constrained by the FF constraint equations, $\psi_{-}=\Lambda_{-} \psi$ form the two lower components. Thus, the quantum field $\hat{\psi}_{+}$can be constructed using only two-component spinor fields as described in Appendix B3. The same construction is used in the case of quantum fields $\hat{\psi}_{+}$and $\hat{\phi}_{+}$in Sec. III A.

The forms (A1) of $\Lambda_{ \pm}$do not fully define a representation of the algebra $\gamma^{\alpha} \gamma^{\beta}+\gamma^{\beta} \gamma^{\alpha}=2 g^{\alpha \beta}$. A slightly different representation from the $\mathrm{FF}$ one described below was introduced before in the context of FF formulation of QCD in Ref. 36], Sec. II B, see also [8], Sec. IV A. The possibility of representing the Dirac fermions with only two-component spinors when one is not interested in the discrete symmetry of parity, is discussed in [10], p. 221. In the FF of Hamiltonian dynamics, the parity symmetry is dynamical and thus not fully understood in complex theories, due to the lack of precise solutions. In the simple model with interactions limited to the mass mixing, the constraint equations that force $\psi_{-}$to form a complete spinor field in combination with $\psi_{+}$can be conveniently solved using the FF representation of $\gamma$-matrices. The parity symmetry is then exhibited in the spectrum of solutions for states of physical particles.

The IF representation we start from is $(k, l=1,2,3)[9]$

$$
\begin{aligned}
\gamma^{0} & =\left[\begin{array}{cc}
1 & 0 \\
0 & -1
\end{array}\right], \quad \gamma^{k}=\left[\begin{array}{cc}
0 & \sigma^{k} \\
-\sigma^{k} & 0
\end{array}\right] \\
\gamma^{5} & =i \gamma^{0} \gamma^{1} \gamma^{2} \gamma^{3}=\left[\begin{array}{ll}
0 & 1 \\
1 & 0
\end{array}\right] \\
\gamma^{5} \gamma^{0} & =\left[\begin{array}{cc}
0 & -1 \\
1 & 0
\end{array}\right], \quad \gamma^{5} \gamma^{k}=\left[\begin{array}{cc}
-\sigma^{k} & 0 \\
0 & \sigma^{k}
\end{array}\right], \\
\sigma^{\mu \nu} & =\frac{i}{2}\left[\gamma^{\mu}, \gamma^{\nu}\right], \quad \sigma^{k l}=\epsilon^{k l m}\left[\begin{array}{cc}
\sigma^{m} & 0 \\
0 & \sigma^{m}
\end{array}\right] \\
\sigma^{0 k} & =\left[\begin{array}{cc}
0 & i \sigma^{k} \\
i \sigma^{k} & 0
\end{array}\right]=i \alpha^{k}, \\
\Lambda_{ \pm} & =\gamma^{0} \gamma^{ \pm} / 2=\frac{1}{2}\left[\begin{array}{cc}
1 & \pm \sigma^{3} \\
\pm \sigma^{3} & 1
\end{array}\right]
\end{aligned}
$$

Every other choice for the $\gamma$ matrices can be obtained [37] using $\tilde{\gamma}^{\mu}=U^{\dagger} \gamma^{\mu} U$ with $U^{\dagger}=U^{-1}$. The FF representation is obtained by defining a special $U$ that provides 
matrices $\Lambda_{ \pm}$of the form (A1) and at the same time transforms IF spinors in a specific way. This way is identified by performing suitable rotations of the conventional elements in spinor basis for physical fermions at rest.

In the IF representation for $\gamma$-matrices, the basis for constructing spinors of fermions at rest can be chosen in the form

$$
\begin{aligned}
& u_{\uparrow}=\left[\begin{array}{l}
1 \\
0 \\
0 \\
0
\end{array}\right], \quad u_{\downarrow}=\left[\begin{array}{l}
0 \\
1 \\
0 \\
0
\end{array}\right], \\
& v_{\uparrow}=C\left[\begin{array}{l}
\overline{1} \\
0 \\
0 \\
0
\end{array}\right]^{T}, \quad v_{\downarrow}=C\left[\begin{array}{l}
\overline{0} \\
1 \\
0 \\
0
\end{array}\right]^{T},
\end{aligned}
$$

where $C=i \gamma^{2} \gamma^{0}$ is the charge conjugation matrix with properties $C=-C^{-1}=-C^{\dagger}=-C^{T}$. The above definition assumes that $v=C \bar{u}^{T}=i \gamma^{2} u^{*}$. One obtains

$$
v_{\uparrow}=\left[\begin{array}{l}
0 \\
0 \\
0 \\
1
\end{array}\right], \quad v_{\downarrow}=\left[\begin{array}{c}
0 \\
0 \\
-1 \\
0
\end{array}\right] .
$$

Action of the projection matrix $\Lambda_{+}$on these IF spinors yields $u_{+}=\Lambda_{+} u$ and $v_{+}=\Lambda_{+} v$. Adjusting normalization to $u_{+}^{\dagger} u_{+}=v_{+}^{\dagger} v_{+}=1$, one obtains

$$
\begin{aligned}
& u_{\uparrow+}=\frac{1}{\sqrt{2}}\left[\begin{array}{l}
1 \\
0 \\
1 \\
0
\end{array}\right], \quad u_{\downarrow+}=\frac{1}{\sqrt{2}}\left[\begin{array}{c}
0 \\
1 \\
0 \\
-1
\end{array}\right], \\
& v_{\uparrow+}=\frac{1}{\sqrt{2}}\left[\begin{array}{c}
0 \\
-1 \\
0 \\
1
\end{array}\right], \quad v_{\downarrow+}=\frac{1}{\sqrt{2}}\left[\begin{array}{c}
-1 \\
0 \\
-1 \\
0
\end{array}\right] .
\end{aligned}
$$

This result involves only two linearly independent spinor basis elements,

$$
u_{\uparrow+}=-v_{\downarrow+}, \quad u_{\downarrow+}=-v_{\uparrow+},
$$

which can be used as the new elements of spinor basis that are invariant under action of and span the image of $\Lambda_{+}$. Acting on the IF spinors at rest with $\Lambda_{-}$yields

$$
\begin{aligned}
& u_{\uparrow-}=\frac{1}{\sqrt{2}}\left[\begin{array}{c}
1 \\
0 \\
-1 \\
0
\end{array}\right], \quad u_{\downarrow-}=\frac{1}{\sqrt{2}}\left[\begin{array}{l}
0 \\
1 \\
0 \\
1
\end{array}\right], \\
& v_{\uparrow-}=\frac{1}{\sqrt{2}}\left[\begin{array}{l}
0 \\
1 \\
0 \\
1
\end{array}\right], \quad v_{\downarrow-}=\frac{1}{\sqrt{2}}\left[\begin{array}{c}
1 \\
0 \\
-1 \\
0
\end{array}\right] .
\end{aligned}
$$

This result also involves only two linearly independent spinor basis elements,

$$
u_{\uparrow-}=v_{\downarrow-}, \quad u_{\downarrow-}=v_{\uparrow-},
$$

which can be used as the two complementary new elements of spinor basis which are invariant under action of and span the image of $\Lambda_{-}$. The complete new spinor basis is called here the FF basis. Its elements are linear combinations of the canonical basis elements with coefficients that form the four columns of the matrix

$$
S=\frac{1}{\sqrt{2}}\left[\begin{array}{cc}
1 & 1 \\
\sigma^{3} & -\sigma^{3}
\end{array}\right] .
$$

Spinors written in terms of their coefficients in the FF basis are marked with the subscript FF.

If an IF spinor is a superposition of the canonical basis elements with coefficients $a_{i}, i=1,2,3,4$, one can write

$$
\begin{aligned}
u_{I F} & =\left[\begin{array}{c}
a_{1} \\
a_{2} \\
a_{3} \\
a_{4}
\end{array}\right]=\frac{a_{1}+a_{3}}{\sqrt{2}} u_{\uparrow+}+\frac{a_{2}-a_{4}}{\sqrt{2}} u_{\downarrow+} \\
& +\frac{a_{1}-a_{3}}{\sqrt{2}} u_{\uparrow-}+\frac{a_{2}+a_{4}}{\sqrt{2}} u_{\downarrow-} .
\end{aligned}
$$

This means that in the FF basis the spinor components are

$$
u_{F F}=S^{T} u_{I F} .
$$

Since the matrix $S$ is orthogonal, $S^{T}=S^{-1}$, one has $u_{I F}=S u_{F F}$. Therefore, also

$$
S \gamma_{F F} u_{F F}=\gamma_{I F} u_{I F}=\gamma_{I F} S u_{F F}
$$

and

$$
\gamma_{F F}=S^{T} \gamma_{I F} S .
$$

Carrying out the required matrix multiplications, one obtains the following $\mathrm{FF}$ representation of the $\gamma$-matrices:

$$
\begin{aligned}
& \gamma^{0}=\left[\begin{array}{ll}
0 & 1 \\
1 & 0
\end{array}\right], \quad \gamma^{3}=\left[\begin{array}{cc}
0 & -1 \\
1 & 0
\end{array}\right], \\
& \gamma^{1}=\left[\begin{array}{cc}
-i \sigma^{2} & 0 \\
0 & i \sigma^{2}
\end{array}\right], \quad \gamma^{2}=\left[\begin{array}{cc}
i \sigma^{1} & 0 \\
0 & -i \sigma^{1}
\end{array}\right],
\end{aligned}
$$

with $\gamma^{k}$ for $k=1,2,3$ obtained from

$$
\gamma^{k}=\frac{1}{2}\left[\begin{array}{cc}
{\left[\sigma^{k}, \sigma^{3}\right]} & -\left\{\sigma^{k}, \sigma^{3}\right\} \\
\left\{\sigma^{k}, \sigma^{3}\right\} & -\left[\sigma^{k}, \sigma^{3}\right]
\end{array}\right] .
$$

Hence,

$$
\alpha^{1}=\left[\begin{array}{cc}
0 & i \sigma^{2} \\
-i \sigma^{2} & 0
\end{array}\right], \quad \alpha^{2}=\left[\begin{array}{cc}
0 & -i \sigma^{1} \\
i \sigma^{1} & 0
\end{array}\right]
$$

and

$$
\alpha^{3}=\left[\begin{array}{cc}
1 & 0 \\
0 & -1
\end{array}\right] \text {. }
$$

One also obtains

$$
\gamma^{5}=\left[\begin{array}{cc}
\sigma^{3} & 0 \\
0 & -\sigma^{3}
\end{array}\right], \gamma^{5} \gamma^{0}=\left[\begin{array}{cc}
0 & \sigma^{3} \\
-\sigma^{3} & 0
\end{array}\right],
$$




$$
\begin{aligned}
\gamma^{5} \gamma^{\perp} & =\left[\begin{array}{cc}
-\sigma^{\perp} & 0 \\
0 & -\sigma^{\perp}
\end{array}\right], \\
\gamma^{5} \gamma^{3} & =\left[\begin{array}{cc}
0 & -\sigma^{3} \\
-\sigma^{3} & 0
\end{array}\right],
\end{aligned}
$$

and

$$
\begin{aligned}
\sigma^{0 k} & =i \alpha^{k}, \quad \sigma^{12}=\left[\begin{array}{cc}
\sigma^{3} & 0 \\
0 & \sigma^{3}
\end{array}\right], \\
\sigma^{23} & =\left[\begin{array}{cc}
0 & \sigma^{1} \\
\sigma^{1} & 0
\end{array}\right], \quad \sigma^{31}=\left[\begin{array}{cc}
0 & \sigma^{2} \\
\sigma^{2} & 0
\end{array}\right] .
\end{aligned}
$$

Eqs. (2.8) in [36], or (4.6) in [8] define a different representation. For example, the FF matrices $\gamma^{ \pm}$are real instead of imaginary and the roles of $\sigma^{1}$ and $\sigma^{2}$ are changed.

\section{Appendix B: FF construction of spinors}

This Appendix defines the spinors that are useful in constructing the FF quantum fields of fermions and solving constraint equations in the model with mass mixing interactions. The spinors are obtained using the FF little group, which belongs to the 2nd class distinguished by Wigner [4]. The little group preserves the null fourvector $n$ (up to a scale) that defines the front hyperplane in space-time through the condition $n x=x^{+}=0$, where $x$ denotes the co-ordinates of points in space-time. The subgroup of the Poincare group that preserves the front hyperplane is also called the group of kinematical symmetries of the FF of dynamics; the group elements do not depend of interactions.

The construction of spinors adopted here draws on Refs. 38 40]. The resulting notation differs slightly from the one introduced in Ref. [41] in Eq. (A3), due to keeping boost matrices for spinors explicitly the same for fermions and anti-fermions and using the kinematical variable $k_{0}^{+}$ instead of a mass parameter, see Eqs. (B18) and (B19) below.

\section{Spinors corresponding to momentum $k_{0}$}

According to Wigner [4], quantum states of a particle are obtained from one state with some specified kinematical momentum $k_{0}$, by applying to the specified state operators that represent elements of the Poincaré group (we do not discuss discrete transformations). In the FF of quantum theory, in distinction from the IF in which boosts depend on interactions, one can use the FF kinematical subgroup of the Poincaré group to construct a fermion state with arbitrary momentum. This means that the FF allows one to construct the states of moving fermions irrespective of interactions while the IF does not allow for such construction.

Let us introduce two basis states for spin- $1 / 2$ fermions with momentum $k_{0}$ and different spin projections on the $z$-axis. Let the kinematical components of the momentum $k_{0}$ be $k_{0}^{+} \neq 0$ and $k_{0}^{\perp}=0$. The component $k_{0}^{-}$is left unspecified by the kinematics because one needs to know the Hamiltonian $P^{-}$to determine if there exists a preferred value of $k_{0}^{-}$. For free fermions of mass $\mu$, the corresponding $P^{-}$would distinguish $k_{0}^{-}=\mu^{2} / k_{0}^{+}$. It would also be natural to assume $k_{0}^{+}=\mu$ for free fermions at rest with respect to the observer who constructs a theory. However, at the level of defining the quantum fields [10] and before one fully understands implications of the assumed dynamics, it is useful to keep the kinematical quantity $k_{0}^{+}$in the notation. Such notation allows one to separate the kinematical construction of quantum field operators from making assumptions about dynamics.

Let the spinors corresponding to the two selected fermion states have the form

$$
u_{0 s}=S^{T} \sqrt{2 k_{0}^{+}}\left[\begin{array}{c}
\chi_{s} \\
0
\end{array}\right]=\sqrt{k_{0}^{+}}\left[\begin{array}{l}
\chi_{s} \\
\chi_{s}
\end{array}\right],
$$

where the matrix $S$ is defined in Appendix $\mathrm{A}$ in Eq. A17) and $\chi_{s}$ with $s= \pm 1$ is the standard two-component Pauli spinor for states with spin up or down. Namely,

$$
\chi_{1}=\chi_{\uparrow}=\left[\begin{array}{l}
1 \\
0
\end{array}\right], \quad \chi_{-1}=\chi_{\downarrow}=\left[\begin{array}{l}
0 \\
1
\end{array}\right] .
$$

This choice is motivated by the physical meaning of spinors in the IF representation of the $\gamma$-matrices considered in Appendix $\chi_{s}$ corresponds to the spin projection on $z$-axis equal $s \hbar / 2$, irrespective of the value of $k_{0}^{+}$.

Similarly, the spinors for two selected basis states of anti-fermions are assumed to have the form

$$
v_{0 s}=S^{T} \sqrt{2 k_{0}^{+}}\left[\begin{array}{c}
0 \\
\varphi_{s}
\end{array}\right]=\sqrt{k_{0}^{+}}\left[\begin{array}{c}
\sigma^{3} \varphi_{s} \\
-\sigma^{3} \varphi_{s}
\end{array}\right] .
$$

In accordance with Appendix $\mathrm{A}$, when one introduces the two-component spinor for anti-fermions using chargeconjugation matrix $C$, so that

$$
\varphi_{s}=-i \sigma^{2} \chi_{s},
$$

one obtains

$$
v_{0 s}=\sqrt{k_{0}^{+}}\left[\begin{array}{c}
-\sigma^{1} \chi_{s} \\
\sigma^{1} \chi_{s}
\end{array}\right] .
$$

Spinors of fermions with momenta other than $k_{0}$ are obtained using a spinor representation of the FF kinematical symmetries.

\section{Spinors for momenta other than $k_{0}$}

Spinors corresponding to states of fermions with momenta other than $k_{0}$ are obtained by applying a spinor representation of the Lorentz transformations built using 
the FF kinematical Poincaré group generators of boosts along $z$-axis, $-J^{+-} / 2=K^{3}$, and the mixed boostrotations, $J^{+1}=K^{1}+J^{2}$ and $J^{+2}=K^{2}-J^{1}$, e.g., see Refs. 42, 43]. The required spinor transformations correspond to the Lorentz subgroup of matrices $L$ of the form

$$
\begin{aligned}
L\left(a_{+}\right) x & =\left[\begin{array}{cccc}
1 / a_{+} & 0 & 0 & 0 \\
0 & a_{+} & 0 & 0 \\
0 & 0 & 1 & 0 \\
0 & 0 & 0 & 1
\end{array}\right]\left[\begin{array}{l}
x^{-} \\
x^{+} \\
x^{1} \\
x^{2}
\end{array}\right], \\
L\left(a_{\perp}\right) x & =\left[\begin{array}{cccc}
1 & a_{\perp}^{2} & 2 a_{1} & 2 a_{2} \\
0 & 1 & 0 & 0 \\
0 & a_{1} & 1 & 0 \\
0 & a_{2} & 0 & 1
\end{array}\right]\left[\begin{array}{l}
x^{-} \\
x^{+} \\
x^{1} \\
x^{2}
\end{array}\right], \\
a_{+} & =k_{2}^{+} / k_{1}^{+}, \quad a_{\perp}=\left(k_{2}^{\perp}-k_{1}^{\perp}\right) / k_{1}^{+} .
\end{aligned}
$$

A four-vector $k_{1}=\left(k_{1}^{-}, k_{1}^{+}, k_{1}^{\perp}\right)$ with $k_{1}^{-}=\left(\mu^{2}+\right.$ $\left.k_{1}^{\perp 2}\right) / k_{1}^{+}$, is changed by these matrices irrespective of the value of the mass parameter $\mu$ to

$$
\begin{aligned}
L\left(a_{+}\right) k_{1} & =\left[\left(\mu^{2}+k_{1}^{\perp 2}\right) / k_{2}^{+}, k_{2}^{+}, k_{1}^{\perp}\right], \\
L\left(a_{\perp}\right) k_{1} & =\left[\left(\mu^{2}+k_{2}^{\perp 2}\right) / k_{1}^{+}, k_{1}^{+}, k_{2}^{\perp}\right] .
\end{aligned}
$$

To transform spinors, one can use a spinor representation of the matrix

$$
\begin{aligned}
& L\left(a_{+}\right) L\left(a_{\perp}\right) \\
= & {\left[\begin{array}{cccc}
1 / a^{+} & a_{\perp}^{2} / a_{+} & 2 a_{1} / a^{+} & 2 a_{2} / a_{+} \\
0 & a_{+} & 0 & 0 \\
0 & a_{1} & 1 & 0 \\
0 & a_{2} & 0 & 1
\end{array}\right], }
\end{aligned}
$$

which transforms the momentum four-vectors according to

$$
L\left(a_{+}\right) L\left(a_{\perp}\right) k_{1}=\left[\left(\mu^{2}+k_{2}^{\perp 2}\right) / k_{2}^{+}, k_{2}^{+}, k_{2}^{\perp}\right],
$$

no matter what the value of $\mu$ is. The required spinor matrix is

$$
\begin{aligned}
& B\left(k_{2}, k_{1}\right) \\
= & \frac{1}{\sqrt{k_{2}^{+} k_{1}^{+}}}\left[k_{2}^{+} \Lambda_{+}+k_{1}^{+} \Lambda_{-}+\left(k_{2}^{\perp}-k_{1}^{\perp}\right) \alpha^{\perp} \Lambda_{+}\right] .
\end{aligned}
$$

By checking the relations

$$
\begin{aligned}
B\left(k_{3}, k_{2}\right) B\left(k_{2}, k_{1}\right) & =B\left(k_{3}, k_{1}\right), \\
B\left(k_{2}, k_{1}\right) & =\left[B\left(k_{1}, k_{2}\right)\right]^{-1}, \\
B\left(k_{1}, k_{1}\right) & =1
\end{aligned}
$$

one can verify that such spinor matrices form a group, as they must as representatives of elements of a subgroup of the Lorentz group.

By replacing $k_{1}$ and $k_{2}$ in Eq. (B13) by $k_{0}$ and $p$, respectively, and using the FF representation of $\gamma$-matrices defined in Appendix A, one obtains

$$
B\left(p, k_{0}\right)=\frac{1}{\sqrt{p^{+} k_{0}^{+}}}\left[\begin{array}{cc}
p^{+} & 0 \\
-i \sigma^{2} p^{1}+i \sigma^{1} p^{2} & k_{0}^{+}
\end{array}\right] .
$$

The spinors for fermions of momentum $p$, irrespective of mass parameters that may be associated with them in a Hamiltonian, are defined as

$$
\begin{aligned}
& u_{p s}=B\left(p, k_{0}\right) u_{0 s}, \\
& v_{p s}=B\left(p, k_{0}\right) v_{0 s},
\end{aligned}
$$

where the spinors corresponding to $k_{0}$ are given by Eqs. (B1) and (B5). In full detail,

$$
\begin{aligned}
& u_{p s}=\frac{1}{\sqrt{p^{+}}}\left[\begin{array}{c}
p^{+} \\
-i \sigma^{2} p^{1}+i \sigma^{1} p^{2}+k_{0}^{+}
\end{array}\right] \chi_{s}, \\
& v_{p s}=\frac{1}{\sqrt{p^{+}}}\left[\begin{array}{c}
-p^{+} \\
+i \sigma^{2} p^{1}-i \sigma^{1} p^{2}+k_{0}^{+}
\end{array}\right] \chi_{-s} .
\end{aligned}
$$

The spinors satisfy relations

$$
\begin{aligned}
& \sum_{s} u_{p s} \bar{u}_{p s}=\not p+k_{0}^{+}, \\
& \sum_{s} v_{p s} \bar{v}_{p s}=\not p-k_{0}^{+},
\end{aligned}
$$

where

$$
\begin{aligned}
p & =\left(p^{-}, p^{+}, p^{\perp}\right), \\
p^{-} & =\frac{p^{\perp 2}+k_{0}^{+2}}{p^{+}} .
\end{aligned}
$$

For free fermions, one could immediately assume that the kinematical parameter $k_{0}^{+}$equals the physical fermion mass that appears in the free fermion Hamiltonian. In the presence of interactions, it is not known prior to solving the theory how the fermion mass parameter that appears in the Hamiltonian is related to the physical fermion mass. The latter situation is exemplified by the model with mass mixing interactions that is solved using the RGPEP in Sec. III In more complex theories, especially in QCD, which is expected to explain confinement of color, it is important to distinguish between the kinematical quantity $k_{0}^{+}$and any dynamically determined concept of a quark mass $\mu$.

\section{Spinors in quantum fields}

The quantum fermion field

$$
\hat{\psi}(x)=\left[\begin{array}{l}
\hat{\zeta}(x) \\
\hat{\xi}(x)
\end{array}\right]
$$

on the front $x^{+}=0$ can be kinematically composed from their Fourier components using momentum variables $p^{+}$ and $p^{\perp}$. The FF constraint equations in theories of physical interest, including the mass mixing model, cause that the independent fermion degrees of freedom are the Fourier components of the field $\hat{\psi}_{+}=\Lambda_{+} \hat{\psi}$. The field $\hat{\psi}_{-}$ is related to the field $\hat{\psi}_{+}$through the constraints. 
Using the representation of $\gamma$-matrices introduced in Appendix A, one has

$$
\hat{\psi}_{+}(x)=\left[\begin{array}{c}
\hat{\zeta}(x) \\
0
\end{array}\right], \quad \hat{\psi}_{-}(x)=\left[\begin{array}{c}
0 \\
\hat{\xi}(x)
\end{array}\right] .
$$

Acting with $\Lambda_{+}$on the spinors of Eqs. (B20) and (B21), one obtains

$$
\begin{aligned}
& \Lambda_{+} u_{p s}=\sqrt{p^{+}}\left[\begin{array}{c}
\chi_{s} \\
0
\end{array}\right], \\
& \Lambda_{+} v_{p s}=\sqrt{p^{+}}\left[\begin{array}{c}
-\sigma^{1} \chi_{s} \\
0
\end{array}\right] .
\end{aligned}
$$

Using these results, one can write

$$
\hat{\zeta}(x)=\sum_{p s} \sqrt{p^{+}}\left[b_{p s} e^{-i p x}-d_{p s}^{\dagger} e^{i p x} \sigma^{1}\right] \chi_{s},
$$

where

$$
\sum_{p s}=\sum_{s= \pm 1} \int_{-\infty}^{+\infty} \frac{d^{2} p^{\perp}}{(2 \pi)^{2}} \int_{0}^{+\infty} \frac{d p^{+}}{2(2 \pi) p^{+}}
$$

and the operators $b_{p s}$ and $d_{p s}$ annihilate fermions and anti-fermions, respectively. Note that one could use $\left|p^{+}\right|$ instead of $p^{+}$visible in Eqs. (B30) and (B31) because $p^{+}>0$ in these equations.

The non-zero canonical anti-commutation relations at $x^{+}=0$ read

$$
\begin{aligned}
\left\{\hat{\psi}_{+}(x), \hat{\psi}_{+}^{\dagger}\left(x^{\prime}\right)\right\} & =\Lambda_{+}\left\{\hat{\zeta}(x), \hat{\zeta}^{\dagger}\left(x^{\prime}\right)\right\} \\
& =\Lambda_{+} \delta^{3}\left(x-x^{\prime}\right) \\
\left\{b_{p s}, b_{p^{\prime} s^{\prime}}^{\dagger}\right\} & =\left\{d_{p s}, d_{p^{\prime} s^{\prime}}^{\dagger}\right\} \\
& =2 p^{+}(2 \pi)^{3} \delta^{3}\left(p-p^{\prime}\right) \delta_{s s^{\prime}}
\end{aligned}
$$

The fields $\hat{\psi}_{-}$depend on interactions through constraints and generally are not related to $\hat{\psi}_{+}$in any simple way. In the case of a theory of free fermions of mass $\mu$, the Dirac equation

$$
(i \not \partial-\mu) \psi=0
$$

takes the form

$$
i \partial^{-} \psi_{+}+i \partial^{+} \psi_{-}-\left(i \alpha^{\perp} \partial^{\perp}+\beta \mu\right)\left(\psi_{+}+\psi_{-}\right)=0
$$

and

$$
\psi_{-}=\frac{1}{i \partial^{+}}\left(i \alpha^{\perp} \partial^{\perp}+\beta \mu\right) \psi_{+} .
$$

Using Eq. (B26), one has

$$
\hat{\xi}=\frac{1}{i \partial^{+}}\left(\sigma^{2} \partial^{1}-\sigma^{1} \partial^{2}+\mu\right) \hat{\zeta}
$$

and

$$
\begin{aligned}
\hat{\psi}(x) & =\hat{\psi}_{+}(x)+\hat{\psi}_{-}(x) \\
& =\sum_{p s}\left[u_{p s}(\mu) b_{p s} e^{-i p x}+v_{p s}(\mu) d_{p s}^{\dagger} e^{i p x}\right],
\end{aligned}
$$

where the spinors are

$$
u_{p s}(\mu)=\frac{1}{\sqrt{\left|p^{+}\right|}}\left[\begin{array}{c}
p^{+} \\
-i \sigma^{2} p^{1}+i \sigma^{1} p^{2}+\mu
\end{array}\right] \chi_{s},
$$

$$
v_{p s}(\mu)=\frac{1}{\sqrt{\left|p^{+}\right|}}\left[\begin{array}{c}
-p^{+} \\
+i \sigma^{2} p^{1}-i \sigma^{1} p^{2}+\mu
\end{array}\right] \chi_{-s},
$$

and the modulus of $p^{+}$is freely inserted using the condition $p^{+}>0$ in the FF Fourier expansion of fields. These spinors match the ones defined kinematically in Eqs. (B18) and (B19) using the momentum $k_{0}$ and transformations $B\left(p, k_{0}\right)$ of Eq. (B17), if one sets $k_{0}^{+}=\mu$. This result is visible by comparing Eqs. (B41) and (B42) with Eqs. (B20) and (B21), correspondingly.

\section{Spinor matrix elements}

The fermion model with mass mixing involves matrix elements of the form

$$
\bar{u}_{1} \Gamma u_{2}=\bar{u}_{01} \gamma^{0} B^{\dagger}\left(p_{1}, k_{01}\right) \gamma^{0} \Gamma B\left(p_{2}, k_{02}\right) u_{02} .
$$

The subscripts 1 and 2 refer to the spin labels and selected momenta $k_{01}$ and $k_{02}$ for fermions of the type 1 and 2 in construction of their states and the corresponding quantum field operators, respectively. Each of these types can be associated with a mass $\mu$ or $\nu$ in the Hamiltonian. If a theory contains more types of fermions than two, the subscripts 1 and 2 may each be associated with any mass in the Hamiltonian. Since

$$
\gamma^{0} B^{\dagger}\left(p, k_{0}\right) \gamma^{0}=\left[B\left(p, k_{0}\right)\right]^{-1}=B\left(k_{0}, p\right),
$$

the matrix elements can be written as

$$
\bar{u}_{1} \Gamma u_{2}=\bar{u}_{01} B_{\Gamma} u_{02} .
$$

where

$$
B_{\Gamma}=B\left(k_{01}, p_{1}\right) \Gamma B\left(p_{2}, k_{02}\right) .
$$

It is assumed that both $k_{01}^{\perp}$ and $k_{02}^{\perp}$ are 0 and the only non-zero kinematical parameters left are $k_{01}^{+}$and $k_{02}^{+}$.

In the fermion model with its interaction limited to mass mixing, all terms in the FF Hamiltonian density are bilinear in the fields. Therefore, the kinematical momentum variables that appear in the spinor matrix elements 
in the FF Hamiltonian involve one kinematical momentum $p=p_{1}=p_{2}$. This means that the matrix elements that count involve only the matrix

$$
\begin{aligned}
B_{\Gamma} & =B\left(k_{01}, p\right) \Gamma B\left(p, k_{02}\right) \\
& =\frac{1}{p^{+} \sqrt{k_{01}^{+} k_{02}^{+}}}\left[k_{01}^{+} \Lambda_{+}+p^{+} \Lambda_{-}-p^{\perp} \alpha^{\perp} \Lambda_{+}\right] \\
& \times \Gamma\left[p^{+} \Lambda_{+}+k_{02}^{+} \Lambda_{-}+p^{\perp} \alpha^{\perp} \Lambda_{+}\right] .
\end{aligned}
$$

For the matrix element for $\Gamma=\gamma^{+}=2 \gamma^{0} \Lambda_{+}$, one uses

$$
B_{\gamma^{+}}=\frac{p^{+} \gamma^{+}}{\sqrt{k_{01}^{+} k_{02}^{+}}}
$$

and obtains

$$
\begin{aligned}
\bar{u}_{1} \gamma^{+} u_{2} & =\bar{v}_{1} \gamma^{+} v_{2}=2 p^{+} \chi_{1}^{\dagger} \chi_{2} \\
& =2 p^{+} \delta_{s_{1} s_{2}} .
\end{aligned}
$$

These matrix elements do not depend on the kinematical parameters $k_{01}$ and $k_{02}$ used in the FF construction of quantum fields for spin-1/2 fermions.

For comparison, one can observe that the matrix element with $\Gamma=1$, relevant to chiral symmetry, does depend on the details of constructing quantum fields. Namely,

$$
\begin{aligned}
B_{1} & =B\left(k_{01}, p\right) B\left(p, k_{02}\right) \\
& =\frac{1}{\sqrt{k_{01}^{+} k_{02}^{+}}}\left[k_{02}^{+} \Lambda_{-}+k_{01}^{+} \Lambda_{+}\right], \\
\bar{u}_{1} u_{2} & =-\bar{v}_{1} v_{2}=\left(k_{01}^{+}+k_{02}^{+}\right) \delta_{s_{1} s_{2}} .
\end{aligned}
$$

Thus, these matrix elements are sensitive to the values of $k_{01}^{+}$and $k_{02}^{+}$used in constructing states and fields. If one insists on $k_{01}^{+}=\mu$ and $k_{02}^{+}=\nu$, the matrix elements equal $\mu+\nu$ for the same spin projections on $z$-axis of fermions of types 1 and 2 at rest.

Note that the FF $z$-axis is also the direction of motion for a fermion with $\perp$ momentum 0 and + momentum different from its mass. Moreover, the ratio $r=p^{+} / \mu$, where $\mu$ denotes the fermion mass, tells one in which direction the fermion moves: $r>1$ corresponds to motion down and $r<1$ to motion against the $z$-axis. Hence, the same projection $s$ denotes different helicities depending on the ratio $r$. These observations are included here in order to prevent a confusion of the spin projection $s$ with just one value of helicity irrespective of $p^{+}$. For the same reason, the interpretation of $s$ as related to helicity depends on the ratio of $k_{0}^{+}$to $\mu$. When the latter depends on the dynamics, one has to be careful in interpreting $s$ in terms of helicity.

\section{Appendix C: Elements of the RGPEP}

Elements of the renormalization group procedure for effective particles (RGPEP) are summarized below for completeness of the article, following notation adopted in Ref. 1] that treats the mass mixing interactions of bosons in a non-perturbative way. More generally, the RGPEP development can be traced back to the invention of the similarity renormalization group procedure [44, 45] and to the conception of the operator formalism that allows one to calculate effective Hamiltonians without limiting their domain in the Fock space expansion of quantum states, and including interactions that involve various numbers of quanta [46, 47]. Compact expressions for the RGPEP in a perturbative series up to the 4th order in interaction are given in Ref. [48].

Effective particles are introduced through a transformation

$$
\psi_{s}=\mathcal{U}_{s} \psi_{0} \mathcal{U}_{s}^{\dagger}
$$

where $\psi_{s}$ is a quantum field operator built from creation and annihilation operators for effective particles of size $s$ and $\psi_{0}$ is a corresponding quantum field operator built from creation and annihilation operators for bare quanta of a local theory. The creation and annihilation operators are denoted collectively by $q_{s}$ and $q_{0}$, respectively. All kinematical quantum numbers that label operators $q$ are the same on both sides of Eq. (C1). Masses are considered dynamical. Interpretation of $s$ as size is based on the form factors that limit how far off energy shell the interactions can extend. The value $s=0$ corresponds to absence of form factors. For a finite $s$, the effective Hamiltonian is band-diagonal on the energy scale and the band width is $\sim 1 / s$.

A canonical Hamiltonian density is built from fields $\psi_{0}$. A corresponding Hamiltonian is a polynomial $\mathcal{H}_{0}\left(q_{0}\right)$ with coefficients $c_{0}$ that are functions of the quantum numbers labeling operators $q_{0}$. Similarly, $\mathcal{H}_{t}\left(q_{t}\right)$ is defined through its coefficients $c_{t}$. For dimensional reasons, it is convenient to use $t=s^{4}$. The RGPEP starts with the equality

$$
\mathcal{H}_{t}\left(q_{t}\right)=\mathcal{H}_{0}\left(q_{0}\right)
$$

which says that the same dynamics is expressed in terms of different operators for different values of $t$. The initial condition being set at $t=0$, variation of the coefficients $c_{t}$ with $t$ is described by the equation obtained by differentiating both sides of

$$
\mathcal{H}_{t}\left(q_{0}\right)=\mathcal{U}_{t}^{\dagger} \mathcal{H}_{0}\left(q_{0}\right) \mathcal{U}_{t}
$$

with respect to $t$, obtaining

$$
\mathcal{H}_{t}^{\prime}\left(q_{0}\right)=\left[\mathcal{G}_{t}\left(q_{0}\right), \mathcal{H}_{t}\left(q_{0}\right)\right]
$$

where $\mathcal{G}_{t}=-\mathcal{U}_{t}^{\dagger} \mathcal{U}_{t}^{\prime}$ and

$$
\mathcal{U}_{t}=T \exp \left(-\int_{0}^{t} d \tau \mathcal{G}_{\tau}\right)
$$

$T$ denotes ordering in $\tau$. 
The RGPEP generator is defined by

$$
\mathcal{G}_{t}=\left[\mathcal{H}_{f}, \mathcal{H}_{P t}\right],
$$

where $\mathcal{H}_{f}$, called the free Hamiltonian, is the part of $\mathcal{H}_{0}\left(q_{0}\right)$ that does not depend on the coupling constants,

$$
\mathcal{H}_{f}=\sum_{i} p_{i}^{-} q_{0 i}^{\dagger} q_{0 i} .
$$

The subscript $i$ denotes particle species and their quantum numbers. The FF free energy of a particle with mass $m_{i}$ and kinematical momentum components $p_{i}^{+}$and $p_{i}^{\perp}$ is

$$
p_{i}^{-}=\frac{p_{i}^{\perp 2}+m_{i}^{2}}{p_{i}^{+}} .
$$

We shall also consider $\mathcal{H}_{f}$ equal to the entire part of $\mathcal{H}$ of the type $q_{0}^{\dagger} q_{0}$ that includes the effective mass parameters $m_{i}$ that do depend on interactions. The operator $\mathcal{H}_{P t}$ is defined knowing $\mathcal{H}_{t}$,

$$
\mathcal{H}_{t}\left(q_{0}\right)=\sum_{n=2}^{\infty} \sum_{i_{1}, i_{2}, \ldots, i_{n}} c_{t}\left(i_{1}, \ldots, i_{n}\right) q_{0 i_{1}}^{\dagger} \cdots q_{0 i_{n}},
$$

to be

$$
\begin{aligned}
\mathcal{H}_{P t}\left(q_{0}\right) & =\sum_{n=2}^{\infty} \sum_{i_{1}, i_{2}, \ldots, i_{n}} c_{t}\left(i_{1}, \ldots, i_{n}\right) \\
& \times\left(\frac{1}{2} \sum_{k=1}^{n} p_{i_{k}}^{+}\right)^{2} q_{0 i_{1}}^{\dagger} \cdots q_{0 i_{n}} .
\end{aligned}
$$

Thus, $\mathcal{H}_{P t}$ differs from $\mathcal{H}_{t}$ by multiplication of each and every term in it by a square of a total + momentum involved in a term [26, 48]. The multiplication leads to preservation of 7 kinematical symmetries of the FF dynamics in the RGPEP.

In summary, the coefficients $c_{t}$ of products of operators $q_{t}$ in the effective Hamiltonians $\mathcal{H}_{t}\left(q_{t}\right)$, are solutions of the equation

$$
\mathcal{H}_{t}^{\prime}=\left[\left[\mathcal{H}_{f}, \mathcal{H}_{P t}\right], \mathcal{H}_{t}\right],
$$

where all operators are written as polynomials in $q_{0}$ and the initial condition is provided by a regulated canonical Hamiltonian with counterterms. The counterterms are calculated using a condition that for finite $t$ the coefficients $c_{t}$ with finite arguments do not depend on the regularization parameters used in the canonical Hamiltonian [44. There is no need for calculating counterterms in the fermion mass mixing model because the coefficients $c_{t}$ with finite arguments do not depend on regularization in this model. The regularization dependence in the model is limited to an additive constant in $\mathcal{H}_{t}$, which drops out from Eq. (66).

The band-diagonal structure of $\mathcal{H}_{t}$ can be seen using a projector $R$ on a subspace in the Fock space. The projected RGPEP equation for $\mathcal{H}_{R}=R \mathcal{H}_{t} R$,

$$
\mathcal{H}_{R}^{\prime}=\left[\left[\mathcal{H}_{f}, \mathcal{H}_{P R}\right], \mathcal{H}_{R}\right],
$$

implies for constant $\mathcal{H}_{f}$ that

$$
\begin{aligned}
\left(\sum_{m n}\left|\mathcal{H}_{I m n}\right|^{2}\right)^{\prime} & =-2 \sum_{k m}\left(\mathcal{M}_{k m}^{2}-\mathcal{M}_{m k}^{2}\right)^{2}\left|\mathcal{H}_{I k m}\right|^{2} \\
& \leq 0,
\end{aligned}
$$

where $\mathcal{H}_{I}=\mathcal{H}-\mathcal{H}_{f}, \mathcal{H}_{P R}=R \mathcal{H}_{P t} R, \mathcal{M}_{k m}$ denotes an invariant mass of the particles in a state labeled $k$ that are connected through the interaction $\mathcal{H}_{I}$ to the particles in a state labeled $m$, and the matrix elements $\mathcal{H}_{m n}=\langle m|\mathcal{H}| n\rangle$ are evaluated in the basis built from eigenstates $|m\rangle$ of $\mathcal{H}_{f}$. According to Eq. (C13), the sum of moduli squared of the interaction Hamiltonian matrix elements decreases as $t$ increases until all off-diagonal matrix elements of the interaction Hamiltonian between states with different free invariant masses vanish. The width of the narrow invariant-mass band in $\mathcal{H}_{R}$ is $s^{-1}$. When the masses in $\mathcal{H}_{f}$ increase with $t$, they reduce the right-hand side of Eq. (C13) to more negative values and thus accelerate formation of the band-diagonal structure of $\mathcal{H}_{t}$.

\section{Appendix D: Solving the RGPEP equations}

It is convenient to define new variables

$$
\begin{aligned}
& \alpha=\mu_{t}^{2} / \delta \mu^{2}, \\
& \beta=\nu_{t}^{2} / \delta \mu^{2}, \\
& \gamma=m_{t}^{2} / \delta \mu^{2},
\end{aligned}
$$

in terms of which Eqs. (85), (866), and (87), read

$$
\begin{aligned}
\alpha^{\prime} & =2 \gamma^{2}, \\
\beta^{\prime} & =-2 \gamma^{2}, \\
\gamma^{\prime} & =-(\alpha-\beta) \gamma,
\end{aligned}
$$

where prime denotes differentiation with respect to the dimensionless parameter $u=\delta \mu^{4} t$ and $\delta \mu^{2}=\mu^{2}-\nu^{2}$. Solutions can be found using the same method as in Ref. [1] because the equations are identical. We quote only key details here, for completeness.

Eqs. (D4) and (D5) imply that $\alpha+\beta$ is a constant and

$$
\begin{aligned}
\delta^{\prime} & =4 \gamma^{2}, \\
\gamma^{\prime} & =-\delta \gamma,
\end{aligned}
$$

where $\delta=\alpha-\beta$. Multiplying these equations by $2 \delta$ and $2 \gamma$, respectively, one arrives at

$$
\begin{aligned}
\delta^{2^{\prime}} & =8 \delta \gamma^{2}, \\
\gamma^{2^{\prime}} & =-2 \delta \gamma^{2},
\end{aligned}
$$

which implies constant

$$
\epsilon^{2}=\delta^{2}+4 \gamma^{2}=\mathcal{T}^{2}-4 \mathcal{D},
$$


where $\mathcal{D}=D / \delta \mu^{4}, \mathcal{T}=T / \delta \mu^{2}, D$ is the determinant and $T$ is the trace of $M^{2}$. Eliminating $\gamma^{2}$ from Eq. (D9), one obtains an ordinary differential equation

$$
\delta^{\prime}=\epsilon^{2}-\delta^{2} .
$$

Integrations of Eqs. (D12) and then (D8), using initial conditions of Eqs. (71), (72), and (73), produce

$$
\begin{aligned}
\mu_{t}^{2} & =m^{2}+\frac{1}{2}\left(\mu^{2}+\nu^{2}\right)+\frac{1}{2} \delta \mu_{t}^{2}, \\
\nu_{t}^{2} & =m^{2}+\frac{1}{2}\left(\mu^{2}+\nu^{2}\right)-\frac{1}{2} \delta \mu_{t}^{2}, \\
\delta \mu_{t}^{2} & =\delta \mu^{2} \frac{\cosh x_{t}+\epsilon \sinh x_{t}}{\cosh x_{t}+\epsilon^{-1} \sinh x_{t}}, \\
m_{t}^{2} & =\frac{m(\mu+\nu)}{\cosh x_{t}+\epsilon^{-1} \sinh x_{t}},
\end{aligned}
$$

where $x_{t}=\epsilon \delta \mu^{4} t$, and $\epsilon=\sqrt{1+[2 m /(\mu-\nu)]^{2}}$ as in Eq. (32).

The RGPEP produces a family of Hamiltonians $\hat{P}_{t}^{-}\left(b_{t \zeta}, d_{t \zeta}, b_{t \omega}, d_{t \omega}\right)$ for $t \geq 0$, members of which are obtained from $\mathcal{P}_{t}^{-}\left(b_{\zeta}, d_{\zeta}, b_{\omega}, d_{\omega}\right)$ in Eq. (67) by replacing operators $q_{p s}$, i.e., $b_{\zeta p s}, d_{\zeta p s}, b_{\omega p s}, d_{\omega p s}$ and their hermitian conjugates, by $q_{t p s}$, i.e.,

$$
\begin{aligned}
b_{t \zeta p s} & =\mathcal{U}_{t} b_{\zeta p s} \mathcal{U}_{t}^{\dagger}, \\
d_{t \zeta p s} & =\mathcal{U}_{t} d_{\zeta p s} \mathcal{U}_{t}^{\dagger}, \\
b_{t \omega p s} & =\mathcal{U}_{t} b_{\omega p s} \mathcal{U}_{t}^{\dagger}, \\
d_{t \omega p s} & =\mathcal{U}_{t} d_{\omega p s} \mathcal{U}_{t}^{\dagger}
\end{aligned}
$$

and their conjugates, correspondingly. In fact, all members of the entire family are the same, see Eq. (C2). The operator $\mathcal{U}_{t}$ is given by Eq. (C5), as a solution of

$$
\mathcal{U}_{t}^{\prime}=-\mathcal{U}_{t}\left[\mathcal{P}_{f}^{-}, \mathcal{P}_{P t}^{-}\right]
$$

Results of Sec. IIIB, in particular Eq. (76) and the fact that the RGPEP respects the FF kinematical symmetries, imply

$$
\begin{aligned}
{\left[\mathcal{P}_{f}^{-}, \mathcal{P}_{P t}^{-}\right] } & =\delta \mu^{2} m_{t}^{2} \mathcal{A} \\
\mathcal{A} & =\int[p]\left(b_{\zeta p s}^{\dagger} b_{\omega p s}-b_{\omega p s}^{\dagger} b_{\zeta p s}\right. \\
& \left.+d_{\zeta p s}^{\dagger} d_{\omega p s}-d_{\omega p s}^{\dagger} d_{\zeta p s}\right)
\end{aligned}
$$

which is a product of the numerical factor that depends on $t$ and a constant operator. Therefore,

$$
\mathcal{U}_{t}=\exp \left(\varphi_{t} \mathcal{A}\right)
$$

where

$$
\varphi_{t}=\arctan \sqrt{\frac{\epsilon+1}{\epsilon-1}}-\arctan e^{x_{t}} \sqrt{\frac{\epsilon+1}{\epsilon-1}} .
$$

To evaluate

$$
q_{t p s}=e^{\varphi_{t} \mathcal{A}} q_{p s} e^{-\varphi_{t} \mathcal{A}}
$$

one can use

$$
\begin{aligned}
& {\left[\mathcal{A}, b_{\zeta p s}\right]=-b_{\omega p s},} \\
& {\left[\mathcal{A}, b_{\omega p s}\right]=b_{\zeta p s},} \\
& {\left[\mathcal{A}, d_{\zeta p s}\right]=-d_{\omega p s},} \\
& {\left[\mathcal{A}, d_{\omega p s}\right]=d_{\zeta p s}}
\end{aligned}
$$

and their hermitian conjugates. There exist combinations

$$
\begin{aligned}
b_{p s} & =b_{\zeta p s}+z b_{\omega p s}, \\
d_{p s} & =d_{\zeta p s}+z d_{\omega p s}
\end{aligned}
$$

for which one has

$$
\begin{aligned}
& {\left[\mathcal{A}, b_{p s}\right]=z b_{p s},} \\
& {\left[\mathcal{A}, d_{p s}\right]=z d_{p s},}
\end{aligned}
$$

if $z^{2}=-1$. One can use $z= \pm i$ for fermions and antifermions equally. Denoting

$$
q_{p s \pm}=q_{\zeta p s} \pm i q_{\omega p s}
$$

one obtains

$$
e^{\varphi_{t} \mathcal{A}} q_{p s \pm} e^{-\varphi_{t} \mathcal{A}}=e^{ \pm i \varphi_{t}} q_{p s \pm} .
$$

Using

$$
\begin{aligned}
b_{\zeta p s} & =\frac{1}{2}\left(b_{p s+}+b_{p s-}\right), \\
d_{\zeta p s} & =\frac{1}{2}\left(d_{p s+}+d_{p s-}\right), \\
b_{\omega p s} & =\frac{-i}{2}\left(b_{p s+}-b_{p s-}\right), \\
d_{\omega p s} & =\frac{-i}{2}\left(d_{p s+}-d_{p s-}\right),
\end{aligned}
$$

one obtains

$$
\begin{aligned}
b_{t \zeta p s} & =\cos \varphi_{t} b_{\zeta p s}-\sin \varphi_{t} b_{\omega p s} \\
d_{t \zeta p s} & =\cos \varphi_{t} d_{\zeta p s}-\sin \varphi_{t} d_{\omega p s} \\
b_{t \omega p s} & =\sin \varphi_{t} b_{\zeta p s}+\cos \varphi_{t} b_{\omega p s} \\
d_{t \omega p s} & =\sin \varphi_{t} d_{\zeta p s}+\cos \varphi_{t} d_{\omega p s}
\end{aligned}
$$

These equations provide explicit definitions of annihilation operators for effective particles corresponding to the RGPEP parameter $t=s^{4}$. The corresponding relations for creation operators are obtained by hermitian conjugation. The inverse relations read

$$
\begin{aligned}
b_{\zeta p s} & =\cos \varphi_{t} b_{t \zeta p s}+\sin \varphi_{t} b_{t \omega p s}, \\
d_{\zeta p s} & =\cos \varphi_{t} d_{t \zeta p s}+\sin \varphi_{t} d_{t \omega p s} \\
b_{\omega p s} & =-\sin \varphi_{t} b_{t \zeta p s}+\cos \varphi_{t} b_{t \omega p s} \\
d_{\omega p s} & =-\sin \varphi_{t} d_{t \zeta p s}+\cos \varphi_{t} d_{t \omega p s} .
\end{aligned}
$$

When $t \rightarrow \infty$, one obtains

$$
\begin{aligned}
\mu_{\infty}^{2} & =m_{1}^{2}, \\
\nu_{\infty}^{2} & =m_{2}^{2}, \\
m_{\infty}^{2} & =0,
\end{aligned}
$$


where $m_{1}$ and $m_{2}$ are the fermion eigenvalue masses of Eq. (30), and

$$
\lim _{t \rightarrow \infty} \varphi_{t}=\varphi
$$

where $\varphi$ is the angle found in Eq. (33). Thus the RGPEP produces a FF quantum Hamiltonian for the two species of free fermions that were obtained in Sec. IIC in the IF of a theory at the price of re-quantization. No such re-quantization is required in the RGPEP.
[1] S. D. Głazek, Phys. Rev. D 85, 125018 (2012).

[2] P. A. M. Dirac, Rev. Mod. Phys. 21, 392 (1949).

[3] P. A. M. Dirac, Phys. Rev. 139, B 684 (1965).

[4] E. P. Wigner, Ann. of Math. 40, 149 (1938).

[5] W. Heisenberg, W. Pauli, Z. f. Phys. 56, 1 (1929).

[6] W. Heisenberg, W. Pauli, Z. f. Phys. 59, 168 (1930).

[7] P. A. M. Dirac, in The Mathematical Foundations of Quantum Theory, Ed. A. R. Marlow (Academic Press, 1978), pp. 1-8.

[8] K. G. Wilson et al., Phys. Rev. D49, 6720 (1994).

[9] J. D. Bjorken, S. D. Drell, Relativistic Quantum Mechanics (McGraw-Hill, 1965).

[10] S. Weinberg, The Quantum Theory of Fields, Vol. I (Cambridge, 1995).

[11] M. E. Peskin, D. V. Schroeder, An Introduction to Quantum Field Theory (Addison-Wesley, 1995).

[12] Y. Nambu, G. Jona-Lasinio, Phys. Rev. 122, 345 (1961).

[13] M. Gell-Mann, R. J. Oakes, B. Renner, Phys. Rev. 175, 2195 (1968).

[14] G. 't Hooft and M. Veltman, Nucl. Phys. B 44, 189 (1972).

[15] J. B. Kogut, L. Susskind, Phys. Rept. C 8, 75 (1973).

[16] A. Casher and L. Susskind, Phys. Rev. D 9, 436 (1974).

[17] K. G. Wilson, Phys. Rev. D 10, 2445 (1974).

[18] M. A. Shifman, A.I. Vainshtein, V. I. Zakharov, Nucl. Phys. B 147, 448 (1979).

[19] R. P. Feynman, Nucl. Phys. B 188, 479 (1981).

[20] E. Witten, Nucl. Phys. B 185, 513 (1981).

[21] J. Gasser, H. Leutwyler, Ann. Phys. 158, 142 (1984).

[22] S. D. Głazek, Phys. Rev. D 38, 3277 (1988).

[23] S. Weinberg, Rev. Mod. Phys. 61, 1 (1989).

[24] P. Maris, C. D. Roberts, P. C. Tandy, Phys. Lett. B 420, 267 (1998).

[25] S. J. Brodsky, H.-C. Pauli, S. S. Pinsky, Phys. Rept. 301, 299 (1998).

[26] S. D. Głazek, Acta Phys. Pol. B 42, 1933 (2011).

[27] S. Weinberg, Phys. Rev. D 83, 063508 (2011).
[28] S. J. Brodsky, C. D. Roberts, R. Shrock, P. C. Tandy, Phys. Rev. C 85, 065202 (2012).

[29] S.-J. Chang, R. G. Root, T.-M. Yan, Phys. Rev. D 7, 1133 (1973).

[30] S.-J. Chang, T.-M. Yan, Phys. Rev. D 7, 1147 (1973).

[31] F. Wegner, Ann. Phys. (Leipzig) 3, 77 (1994).

[32] F. Wegner, J. Phys. A: Math. Gen. 39, 8221 (2006).

[33] S. K. Kehrein, The Flow Equation Approach to ManyParticle Systems, (Springer, 2006).

[34] S. D. Głazek, A. P. Trawiński, Phys. Rev. D 87, 025002 (2013).

[35] P. A. M. Dirac, Proc. Roy. Soc. (London) A 117, 610 (1928).

[36] W.-M. Zhang, A. Harindranath, Phys. Rev. D 48, 4881 (1993).

[37] E.g., see R. H. Good, Jr., Rev. Mod. Phys. 27, 187 (1955), and refs. therein.

[38] S. D. Głazek, The Weinberg equation with spin, M. Sc. Diploma thesis, University of Warsaw, 1980.

[39] S. D. Głazek, The connection between the light front $d y$ namics and the infinite momentum frame, University of Warsaw preprint No. IFT/15/82.

[40] S. D. Głazek, Acta Phys. Pol. B 15, 889 (1984).

[41] G. P. Lepage, S. J. Brodsky, Phys. Rev. D 22, 2157 (1980).

[42] H. Leutwyler, J. Stern, Ann. Phys. 112, 94 (1978).

[43] H. Leutwyler, J. Stern, Phys. Lett. B 73, 75 (1978).

[44] S. D. Głazek, K. G. Wilson, Phys. Rev. D 48, 5863 (1993).

[45] S. D. Głazek, K. G. Wilson, Phys. Rev. D 49, 4214 (1994).

[46] S. D. Głazek, Similarity Renormalization Scheme for Hamiltonians, in "Theory of hadrons and light-front QCD" (World Scientific, Singapore 1995).

[47] S. D. Głazek, Acta Phys. Pol. B 29, 1979 (1998).

[48] S. D. Głazek, Acta Phys. Pol. B 43, 1843 (2012). 